\title{
CLOSE ISOTOPIES ON PIECEWISE-LINEAR MANIFOLDS
}

\author{
BY \\ RICHARD T. MILLER( $\left.{ }^{(}\right)$
}

We say that two maps of a set into a metric space are within $\delta$ everywhere if, for each point in the set, its images under the maps are within $\delta$ of each other.

Let $I=[-1,1]$. We prove the following theorems.

THEOREM 9. For $q-n \geqq 3$ and $\varepsilon>0$, there is $a \delta>0$ such that if $f: I^{n} \rightarrow I^{q}$ is a proper piecewise-linear embedding with $f \mid \mathrm{bdy}\left(I^{n}\right)$ the identity and $f$ within $\delta$ of the identity everywhere, then there is a piecewise-linear ambient isotopy of $I^{q}$ that fixes bdy $\left(I^{q}\right)$, that moves points less than $\varepsilon$, and that takes $f$ to the identity.

THEOREM 11. Let $M$ be a compact piecewise-linear n-manifold, let $\hat{M}$ be a compact piecewise-linear $n$-submanifold of $M$, let $Q$ be a piecewise-linear q-manifold, and let $n \leqq q-3$. Suppose $g: M \rightarrow \operatorname{int}(Q)$ is a piecewise-linear embedding. Let $N$ be a neighborhood of

$$
g(\overline{M-\hat{M}})
$$

in $Q$. Then for $\varepsilon>0$, there is $a \delta>0$ such that if $h: M \rightarrow \operatorname{int}(Q)$ is a piecewiselinear embedding with $h|\hat{M}=g| \hat{M}$, and with $h$ within $\delta$ of $g$ everywhere, then there is a piecewise-linear ambient isotopy of $Q$ that is fixed outside $N$, that moves points less than $\varepsilon$, and that takes $h$ to $g$.

THEOREM 14. Let $M$ be a compact piecewise-linear $n$-manifold, let $\hat{M}$ be a compact piecewise-linear $n$-submanifold, and let $Q$ be a piecewise-linear $q$-manifold. Suppose $n \leqq q-3$ and $q \geqq 5$. Suppose $g: M \rightarrow$ int $(Q)$ is a locally-flat embedding such that $g \mid \hat{M}$ is piecewise-linear. Let $N$ be a neighborhood of

$$
g(\widehat{M-\hat{M}})
$$

in $Q$. Then for $\varepsilon>0$, there is an ambient isotopy of $Q$ that is fixed outside $N$, that moves points less than $\varepsilon$, and that takes $g$ to a piecewise-linear embedding of $M$ in $Q$.

The problem underlying Theorems 9 and 11 is the following. Suppose $f: I^{n} \rightarrow I^{q}$ is a proper p.l. embedding that is the identity on bdy $I^{n}$. In general, $f\left(I^{n}\right) \cap$ $\left(\{0\} \times I^{q-1}\right)$ is just an $n$-complex. If the vertices of $f\left(I^{n}\right)$ are moved just enough to get them off $\{0\} \times I^{q-1}$ but not enough to alter the fact that $f$ is an embedding, then

Received by the editors January 30, 1969.

(1) Much of this work is contained in the author's Ph.D. thesis at the University of Michigan. The author wishes to thank Professor James Kister for his help and encouragement during the preparation of this paper.

Copyright (C) 1970, American Mathematical Society 
the new $f\left(I^{n}\right) \cap\left(\{0\} \times I^{q-1}\right)$ is a p.l. (n-1)-submanifold of both $f\left(I^{n}\right)$ and $\{0\}$ $\times I^{q-1}$. A vertex move like that described above may actually be accomplished by an arbitrarily small ambient isotopy of $I^{q}$. We would like to do still better and make the intersection an ( $n-1)$-cell. If $n \leqq q-3$, Zeeman's unknotting theorem says there is an ambient isotopy of $I^{q}$ that moves $f$ to the identity (and incidentally therefore makes $f\left(I^{n}\right) \cap\left(\{0\} \times I^{q-1}\right)$ the $(n-1)$-cell $\left.\{0\} \times I^{n-1}\right)$. But there is no reason to suspect that this ambient isotopy is small even if $f$ is close to the identity. In fact the ambient isotopy actually constructed necessarily moves points a long way. Below we outline a construction that yields a small ambient isotopy if the original embedding $f$ is close to the identity.

Think of the last coordinate of a point in $I^{q}$ as its height. Thus, $I^{q-1} \times\{1\}$ and $I^{q-1} \times\{-1\}$ are respectively the "top" and "bottom" of $I^{q}$. A point $(x, s)$ is above $(x, t)$ if $x \in I^{q-1}$ and $s>t$ are in $I$. If $K$ is a complex in $I^{q}$, the shadow set $K_{s}$ of $K$ is $K$ together with all points below $K$.

Suppose that $K$ is a subcomplex of $f\left(I^{n}\right)$ that is also contained in $[-1,0] \times I^{q-1}$. We define the subset $W_{K}$ of $I^{q}$ as

$$
\left(\text { bdy }\left([-1,0] \times I^{q-1}\right)-\left(\{0\} \times \operatorname{int} I^{q-1}\right)\right) \cup K_{S} \text {. }
$$

Then there is a collapse of $[-1,0] \times I^{q-1}$ onto $W_{K}$ given by collapsing in a shelf just under the top of $I^{q}$ and then collapsing downward until stopped by either $K$ or the bottom of $I^{q}$. (See Figure 1.) Since $f$ is a proper embedding, a sufficiently low shelf will not hit $K$, and consequently will not hit $K_{S}$. By "isotopy following collapse" (Proposition 1), there is an ambient isotopy of $I^{q}$ fixing bdy $I^{q}$ that takes $N\left([-1,0] \times I^{q-1}, I^{q^{\prime \prime}}\right)$ (the second derived neighborhood of $[-1,0] \times I^{q-1}$ in $\left.I^{q}\right)$ onto $N\left(W_{K}, I^{q^{\prime \prime}}\right)$. It is important in the sequel that the ambient isotopy follows the collapse closely in the sense of Proposition 1. The upshot of this discussion is that it suffices for us to arrange that the intersection of $f\left(I^{n}\right)$ with $N\left(W_{K}, I^{q^{\prime \prime}}\right)$ have nice properties (this is done by judicious choice of $K$ ), since then if we run this ambient isotopy following collapse backward, the intersection of the isotoped $f\left(I^{n}\right)$ with $N\left([-1,0] \times I^{q-1}, I^{q^{\prime \prime}}\right)$ will have the same nice properties. This will lead quickly to a small move that makes the twice moved $f\left(I^{n}\right) \cap$ $\left(\{0\} \times I^{q-1}\right)$ nice.

We come to the question of choosing $K$. First note that no matter which $K$ we pick, $N\left(K, f\left(I^{n}\right)^{\prime \prime}\right)$ is contained in $N\left(W_{K}, I^{q^{\prime \prime}}\right)$. If a simplex of $f\left(I^{n}\right)-K$ meets $K_{S}$ (it cannot meet anything else in $W_{K}$ ), then its second derived neighborhood in $f\left(I^{n}\right)$ lies in $N\left(W_{K}, I^{q^{\prime \prime}}\right)$. Conversely and happily it is easy to see that if no simplex in $f\left(I^{n}\right)-K$ meets $K_{S}$ then the intersection of $f\left(I^{n}\right)$ with $N\left(W_{K}, I^{q^{\prime \prime}}\right)$ is precisely $N\left(K, f\left(I^{n}\right)^{\prime \prime}\right)$. So the condition we need on $K$ is that no simplex of $f\left(I^{n}\right)-K$ lies below a simplex of $K$. In particular, if $f\left(I^{n}\right)$ sunny collapses to $K$ then this condition is satisfied.

We get a second condition on $K$ by requiring that $f\left(I^{n}\right) \cap N\left(W_{K}, I^{q^{\prime \prime}}\right)$ 
$\left(=N\left(K, f\left(I^{n}\right)^{\prime \prime}\right)\right.$ by the first condition $)$ is an $(n-1)$-cell. This happens precisely when $K$ is collapsible.

Thus, if $f\left(I^{n}\right)$ sunny collapses to $K$ and $K$ collapses, then the previous discussion shows that there is an ambient isotopy that fixes bdy $I^{q}$ and moves $f\left(I^{n}\right)$ so that its new intersection with $\{0\} \times I^{q-1}$ is an $(n-1)$-cell.

If we are interested (which we are) in the further question of whether the moved $f\left(I^{n}\right) \cap\left([-1,0] \times I^{q-1}\right)$ is an unknotted $n$-ball in $[-1,0] \times I^{q-1}$, the answer (according to Zeeman) is yes provided the collapse of $K$ to a point is actually sunny. If the original pair $\left\{I^{q}, f\left(I^{n}\right)\right\}$ is also unknotted then we can conclude by standard p.l. theorems that the moved $f\left(I^{n}\right) \cap\left([0,1] \times I^{q-1}\right)$ is unknotted in $[0,1] \times I^{q-1}$.

Until the end of Lemma 12, all maps and manifolds are piecewise-linear unless otherwise noted. Thereafter they are p.l. only when so noted. The diameter of a set is defined as the maximum distance between two points in the set (all our sets are contained in Euclidean $n$-space $\left(R^{n}\right)$ for some $n$ ). If $K$ is a simplicial complex, define the mesh of $K$, written mesh $(K)$, as the maximum diameter of the simplexes in $K$. Let $K^{\prime}$ denote a first derived subdivision of $K$, and let $K^{(r)}$ denote the $r$ th barycentric subdivision of $K$. If $L$ is a subset of $K$, then the neighborhood of $L$ in $K$, written $N(L, K)$, is the subcomplex of $K$ generated by the simplexes of $K$ that intersect $L$. If $\delta$ is a real number greater than or equal to zero, the $\delta$-neighborhood of $L$ in $K$ is defined as the set of points in $K$ whose distance from $L$ is less than or equal to $\delta$. If $S$ is a set, let bdy $(S)$ denote the boundary of $S$, and let int $(S)$ denote the interior of $S$. Let $I_{w}=[-w, w]$. Take $R^{n}$ to be included in $R^{n+1}$ as the hyperplane $R^{n} \times\{0\}$.

If $P$ and $R$ are simplexes and $R$ is a top-dimensional face of $P$, we may think of the collapse of $P$ through $R$ to (bdy $(P)$-int $(R)$ ) as a deformation retraction of $P$ onto (bdy $(P)-$ int $(R)$ ). Consequently, if $Y$ is a simplicial complex, if $X$ is a subcomplex of $Y$, and if $Y$ collapses simplicially to $X$, then the collapse may be regarded as a deformation retraction of $Y$ onto $X$ formed by the composition of the elementary simplicial collapses involved, each being considered a deformation retraction. For each simplex $Z$ in $Y$, define image $\backslash_{Y \backslash X}(Z)$, the image of $Z$ under the collapse from $Y$ to $X$, as the image of $Z$ under the collapse considered as a deformation retraction, and define $\operatorname{track}_{Y \backslash X}(Z)$, the track of $Z$ under the collapse from $Y$ to $X$, as the set of points in $Y$ that are images of $Z$ at some time during the deformation retraction.

Proposition 1. Let $M$ be an $n$-manifold contained in a q-manifold $Q$. Suppose that $n=q$ or $n \leqq q-3$, that int $(M)$ is contained in int $(Q)$, and that $M$ is a subcomplex of $Q$. Let $Y$ and $X$ be subcomplexes of $M$, and let $W$ be a subcomplex of $Q$. If $Y$ collapses simplicially to $X$ and $W \cap(Y-X)=\varnothing$, then there exists an isotopy $\Phi_{t}$ of $N\left(Y, M^{\prime \prime}\right)$ into itself such that $\Phi_{1}\left(N\left(Y, M^{\prime \prime}\right)\right)=N\left(X, M^{\prime \prime}\right)$, such that

$$
\Phi_{t} \mid N\left(W, M^{\prime \prime \prime}\right) \cup\left(M-N\left(N\left(\text { vertices in }(Y-X)^{\prime}, M^{\prime \prime}\right), M^{\prime \prime}\right)\right)
$$


is the identity, and such that if $Z$ is a subcomplex of $Y$, then

$$
\Phi_{1}\left(N\left(Z, M^{\prime \prime}\right)\right) \subset N\left(\underset{Y \backslash X}{\operatorname{image}}(Z), M^{\prime \prime}\right) \text {. }
$$

If, in addition, $(Y-X)$ is contained in the interior of $Q$, then $\Phi_{t}$ extends to an ambient isotopy of $Q$ (also called $\Phi_{t}$ ) for which

and

$$
\Phi_{t}\left(N\left(N\left(Z, Q^{\prime \prime}\right), Q^{m}\right)\right) \subset N\left(N\left(\underset{Y \searrow X}{\operatorname{track}}(Z), Q^{\prime \prime}\right), Q^{\prime \prime}\right)
$$

$$
\Phi_{t} \mid N\left(W, Q^{\prime \prime}\right) \cup\left(Q-N\left(N\left(\text { vertices in }(Y-X)^{\prime}, Q^{\prime \prime}\right), Q^{\prime \prime}\right)\right)
$$

is the identity.

Proposition 1 is a convenient tool for constructing isotopies and ambient isotopies that behave in a reasonably predictable manner. Unfortunately, our proof is rather long, and we omit it here. It is reproduced in full in [8].

Define the diameter of the simplicial collapse from $Y$ to $X$ as the maximum diameter of $\operatorname{track}_{Y \backslash X}(Z)$ for simplexes $Z$ in $Y$.

COROLlary 2. If the mesh of the triangulation of $Q$ is less than $\delta$, if $(Y-X)$ is contained in the interior of $Q$, and if the diameter of the collapse from $Y$ to $X$ is less than $\gamma$, then the ambient isotopy $\Phi_{t}$ moves points less than $(\gamma+2 \delta)$.

Proof. For each simplex $Z$ in $Y$, the diameter of $\operatorname{track}_{Y \backslash X}(Z)$ is less than $\gamma$. In addition,

$$
\begin{aligned}
\Phi_{t}\left(N\left(N\left(\underset{Y \backslash X}{\operatorname{track}}(Z), Q^{\prime \prime}\right), Q^{\prime \prime}\right)\right) & \subset N\left(N\left(\underset{Y \backslash X}{\operatorname{track}}(Z), Q^{\prime \prime}\right), Q^{\prime \prime \prime}\right) \\
& \subset N\left(\underset{Y \backslash x}{\operatorname{track}}(Z), Q^{\prime}\right) .
\end{aligned}
$$

The last set has diameter less than $(\gamma+2 \delta)$. Thus, points in $N\left(N\left(Y, Q^{\prime \prime}\right), Q^{\prime \prime}\right)$ are moved less than $(\gamma+2 \delta)$. Points in

are fixed.

$$
\left(Q-N\left(N\left(Y, Q^{\prime \prime}\right), Q^{\prime \prime}\right)\right)
$$

We now deal with the problem of taking an embedding $f$ of $I^{n}$ in $I^{q}$ that is close to the identity and sunny collapsing $f\left(I^{n}\right)$ to a subcomplex $K$ that comprises about the left-hand half of $f\left(I^{n}\right)$, and then sunny collapsing this complex to a point. The general philosophy here is that if we can cut $f\left(I^{n}\right)$ approximately in half, we can cut it into many approximately equal sized pieces (but not so many that the characteristic size of each piece is small relative to the closeness of the embedding $f$ ).

To achieve our sunny collapses we slightly modify Zeeman's method of sunny collapsing $f\left(I^{n}\right)$ to a point. In particular, we choose a sequence of cones, each having one less dimension than and contained in the preceding one, but not exactly as a subcone. The first cone is $f\left(I^{n}\right)=f\left(\right.$ bdy $\left.\left(I^{n}\right) \cdot \nu\right)$ where $\nu \in$ int $I^{n}$. The induction hypothesis then requires that the dimension of the subcomplex of each cone that is shadowed by that cone is 2 or more less than the dimension of the cone. (Enter the condition $n \leqq q-3$.) This means that if we have already collapsed to the cone of dimension $j$ then all the $j$ and $j-1$ simplexes of the cone are un- 
shadowed and so any collapse of $j$-dimensional simplexes is sunny. Unfortunately, in order to have the induction hypothesis satisfied for the $(j-1)$ dimensional cone we had to choose one that could not be collapsed to by only $j$-dimensional collapses. It is actually necessary to collapse out a few $(j-1)$ simplexes through $(j-2)$ dimensional faces that shadow one another. These can be sunny collapsed if we do the collpses in the correct order (namely, the ones on top first).

Now, in our modified form we want to leave approximately the left-hand half of $f\left(I^{n}\right)$. Thus, the collapse of the $j$-dimensional cone to the $(j-1)$ dimensional cone must leave most of the $j$-simplexes that lie in the left-hand half. This much goes easily. But recall that there are still $(j-1)$ simplexes that must be collapsed in a specific order. Some of these simplexes may not be free in our case because we left some higher dimensional simplexes (the left-hand ones). Conceivably this could prevent us from collapsing out any of the $(j-1)$ simplexes. This does not happen since the embedding $f$ is close to the identity which implies that only simplexes near the left-hand half can be shadowed by simplexes in the left-hand half. To complete this step we collapse out those $(j-1)$ simplexes that we can and forget about the others. (They are near the left-hand side anyway.) This is the way we collapse $f\left(I^{n}\right)$ to $K$. The collapse of $K$ to a point is done by Zeeman's argument as outlined above. Here we do not have to worry about leaving any simplexes.

Recall that $I=[-1,1]$ and $I_{w}=[-w, w]$. Let $k$ and $q$ be nonnegative integers such that $k<q$. Call the direction of the last coordinate in $I_{2}^{k} \times I^{q-k}$ vertical or sunny, and call the projection

$$
\pi: I_{2}^{k} \times I^{q-k} \rightarrow I_{2}^{k} \times I^{q-k-1}
$$

sunny projection. If two points lie on the same vertical line, say that the one with the smaller last coordinate is shadowed by the other. A set is shadowed by another set if any of its points is shadowed by a point in the other.

If $K$ and $M$ are simplicial complexes, and some subdivision of $K$ is a subcomplex of a subdivision of $M$, then call $K$ a subpolyhedron of $M$. The underlying topological space of a simplicial complex $K$ is denoted by $|K|$.

Let $K$ and $L$ be subpolyhedra of $I_{2}^{k} \times I^{q-k}$ and $K$ collapse to $L$ by an elementary collapse. If $(|K|-|L|)$ is not shadowed by $|K|$, say that the collapse is sunny Call a sequence of elementary sunny collapses a sunny collapse.

Let $S$ be a subset of $I_{2}^{k} \times I^{q-k}$, and denote by $S^{*}$ the singular set of $S$ with respect to sunny projection. Thus $S^{*}$ is the collection of those points in $S$ which shadow or are shadowed by another point in $S$.

Let $n$ be a nonnegative integer such that $q-3 \geqq n \geqq k$, and let $\delta$ be a positive real number such that $4 \delta(n+2)<\frac{1}{2}$. If $k=0$, define

$$
\begin{aligned}
& A=[-1,-4 \delta(n+2)] \times I^{q-1}, \\
& B=[-1,0] \times I^{q-1}, \\
& A_{j}=[-1,-4 \delta(n+1-j)] \times I^{n-1}, \text { and } \\
& \text { the point } \bar{v}=(-1,0, \ldots, 0) .
\end{aligned}
$$


If $k>0$, define

$$
\begin{aligned}
& A=I_{1-4 \delta(n+2)}^{k} \times I^{q-k}, \\
& B=I^{q}, \\
& A_{j}=I_{1-4 \delta(n+1-j)}^{k} \times I^{n-k}, \text { and } \\
& \bar{v}=(0, \ldots, 0) .
\end{aligned}
$$
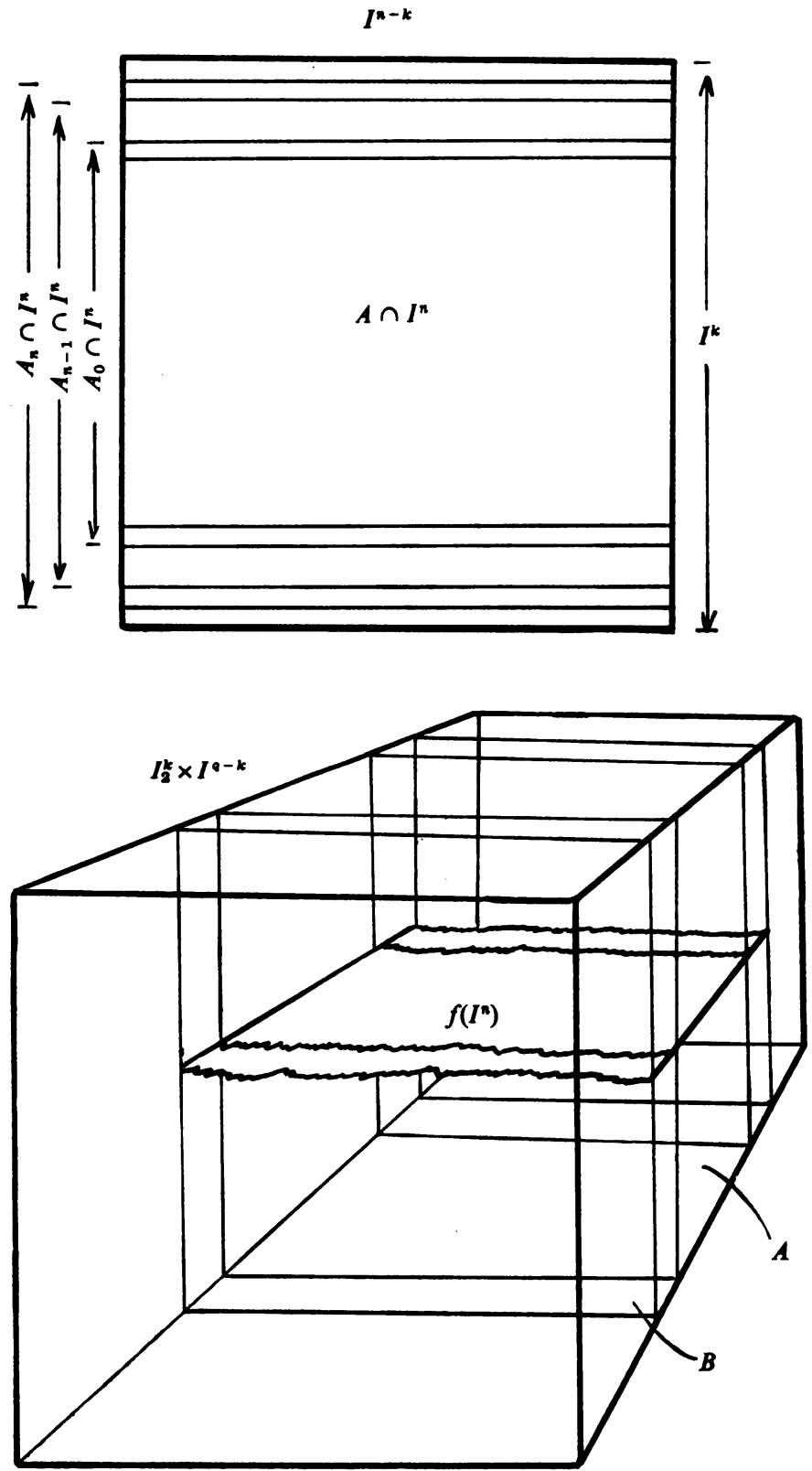

Figure 1 
LemMa 3. Suppose $f: I^{n} \rightarrow I_{2}^{k} \times I^{q-k}$ is an embedding with $f\left(I^{n}\right) \cap \operatorname{bdy}\left(I_{2}^{k} \times I^{q-k}\right)=I^{k} \times$ bdy $\left(I^{n-k}\right)$, $f \mid I^{k} \times$ bdy $\left(I^{n-k}\right)$ the identity, $f$ within $\delta$ of the identity everywhere,
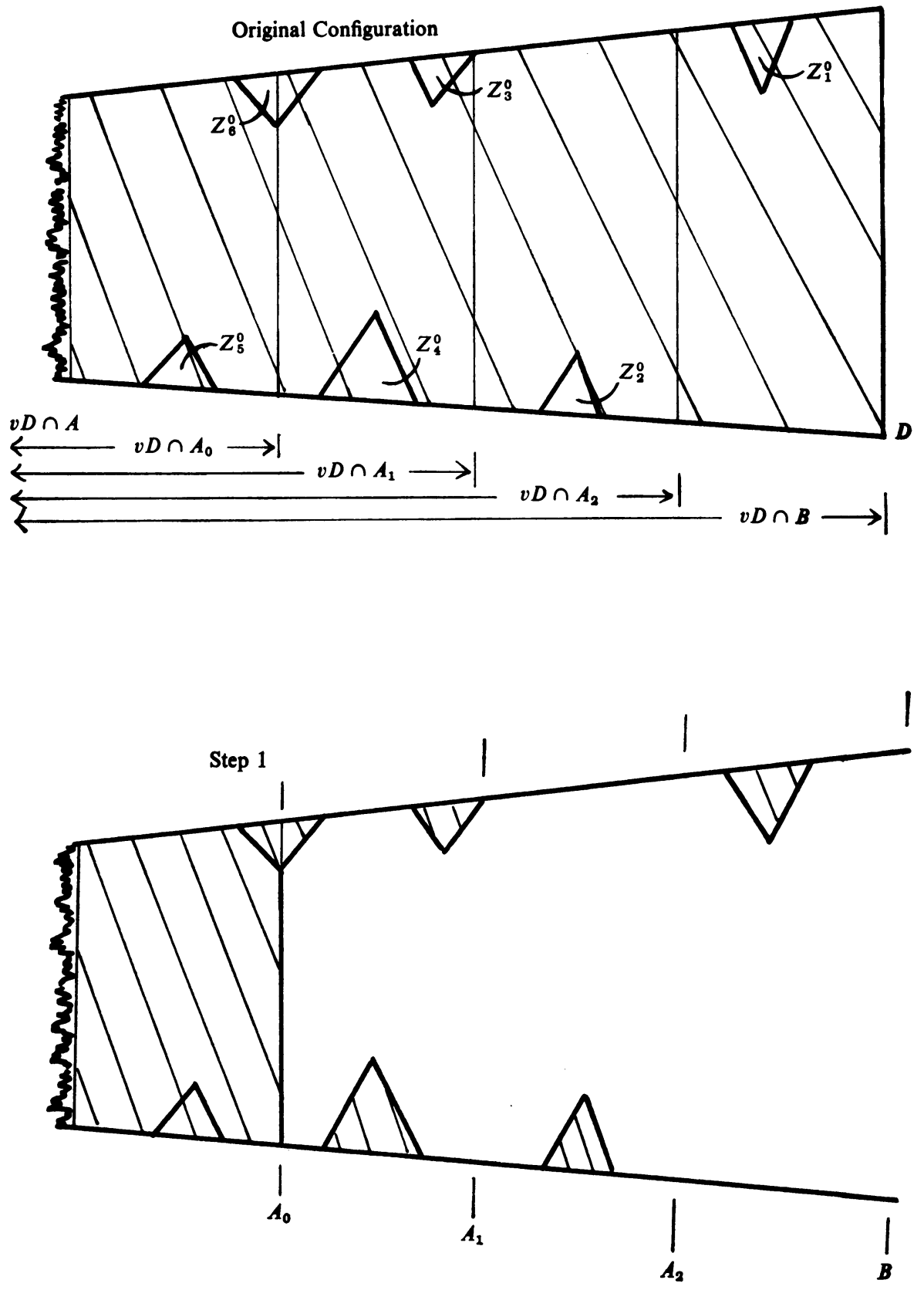

Figure 2. The collapses in Lemma $3(k>0)$. 
$f\left(I^{n}\right)$ meeting every vertical line finitely, and $f\left(I^{n}\right)^{*}$ having dimension $\leqq n-2$.

Then there is a sunny collapse of $f\left(I^{n}\right)$ to a subpolyhedron $\hat{R}$ such that $\hat{K} \supset f\left(I^{n}\right) \cap A$, the set $\hat{R} \subset B$, and there is a sunny collapse of $R$ to a point $f(v)$ near $f(\bar{v})$.
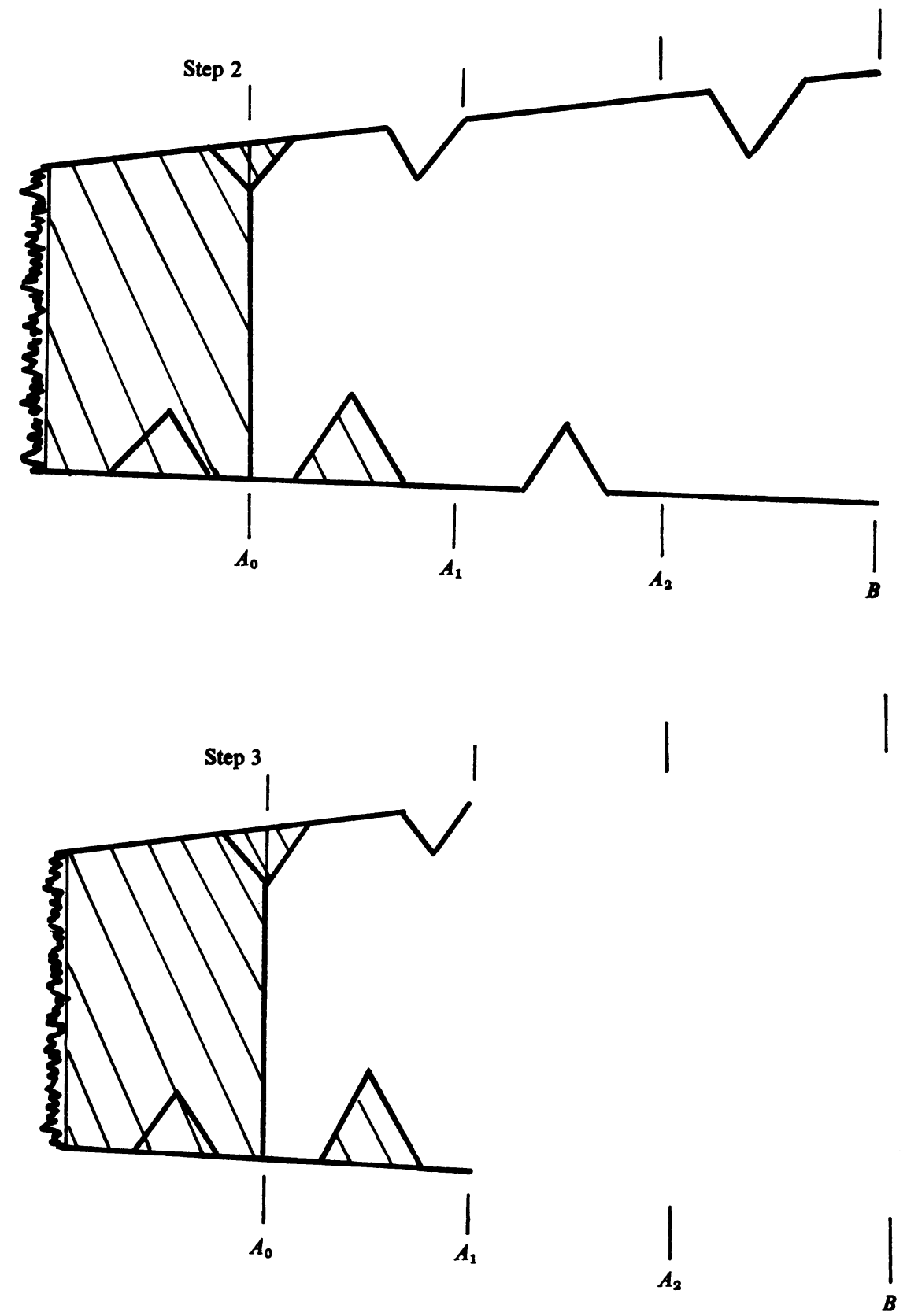

FIGURE 3. Lemma 3. 
Proof. Subdivide $I^{n}$ so that $A_{j}$ is a subcomplex (for $j=-1, \ldots, n+1$ ), and the mesh of the triangulation is less than $\delta$. The first part of the proof is Zeeman's construction in the proof of Lemma 23 except for a modification that is discussed when it is made.

Construct inductively two sequences of polyhedra,

$$
\left.I_{2}^{k} \times I^{q-k} \supset f\left(I^{n}\right)=X_{0} \supset X_{1} \supset \ldots \supset X_{n}=v \text { (= a point }\right)
$$

and

$$
\operatorname{bdy}\left(I^{n}\right)=K^{n-1} \supset K^{n-2} \supset \ldots \supset K^{n-i-1} \supset \ldots \supset K^{-1}=\varnothing,
$$

and (for each $i$ ) a homeomorphism $f_{i}: X_{i} \stackrel{\text { onto }}{\longrightarrow} v K^{n-i-1}$. These sets and maps are to be such that

$f_{i}\left(X_{i}^{*}\right)$ does not contain $v$,

$f_{i}\left(X_{i}^{*}\right)$ meets each generator of the cone $f_{i} X_{i}$ finitely,

dimension $X_{i}^{*} \leqq(n-i-2)$, and

there is a sunny collapse from $X_{i-1}$ to $X_{i}$.

Start with $f_{0}=f^{-1}: f\left(I^{n}\right) \stackrel{\text { onto }}{\longrightarrow} I^{n}$. Since $f\left(I^{n}\right)^{*}$ has dimension $\leqq(n-2)$, there is a point $v \in$ int $\left(I^{n}\right)$ that lies within $\delta$ of $\bar{v}$ and is in general position with respect to $f_{0}\left(X_{0}^{*}\right)\left(=f^{-1}\left(f\left(I^{n}\right)^{*}\right)\right)$. Star $I^{n}$ at $v$. This makes $I^{n}$ into the cone $v\left(\right.$ bdy $\left.\left(I^{n}\right)\right)$ on bdy $\left(I^{n}\right)$.

Next is the inductive step. Suppose $f_{i-1}: X_{i-1} \rightarrow v K^{n-i}$ satisfies the inductive conditions. We can assume $X_{i-1}$ and $v K^{n-i}$ are triangulated so $f_{i}$ is simplicial. Choose a conical subdivision $\left(v K^{n-i}\right)^{\sim}$ of $v K^{n-i}$. This contains the subdivision $\left(K^{n-i}\right)^{\sim}$ of $K^{n-i}$. Define $K^{n-i-1}$ to be the $(n-i-1)$-skeleton of $\left(K^{n-i}\right)^{\sim}$. Notice that $v K^{n-i-1} \subset v K^{n-i}$, and denote the inclusion map by

$$
\text { inc }_{i}: v K^{n-i-1} \rightarrow v K^{n-i} .
$$

Construct another embedding, $e_{i}: v K^{n-i-1} \rightarrow v K^{n-i}$, as follows. Choose subdivisions $\left(f_{i-1}\left(X_{i-1}^{*}\right)\right)^{\sim}$ of $f_{i-1}\left(X_{i-1}^{*}\right)^{\sim}$ and $\left(I_{2}^{k} \times I^{q-k-1}\right)^{\sim}$ of $\left(I_{2}^{k} \times I^{q-k-1}\right)$, and a triangulation $\left(X_{i-1}^{*}\right)^{\sim}$ of $\left|X_{i-1}^{*}\right|$ such that for these choices, the maps

$$
f_{i-1}:\left(X_{i-1}^{*}\right)^{\sim} \rightarrow\left(f_{i-1}\left(X_{i-1}^{*}\right)\right)^{\tilde{\nu}}
$$

and

$$
\pi_{\mid}^{\prime}\left(X_{i-1}^{*}\right)^{\sim}:\left(X_{i-1}^{*}\right)^{\sim} \rightarrow\left(I_{2}^{k} \times I^{q-k-1}\right)^{\sim}
$$

are simplicial. By induction, dimension $\left(X_{i-1}^{*}\right) \leqq(n-i-1)$. If $\sigma_{1}^{i-1}, \sigma_{2}^{i-1}, \ldots, \sigma_{r}^{i-1}$ are the $(n-i-1)$-simplexes of $\left(X_{i-1}^{*}\right)^{\sim}$, the ordering may be chosen so that all the points of $f\left(I^{n}\right)$ which overshadow int $\left(\sigma_{w}^{i-1}\right)$ are contained in $\bigcup_{j<w}$ int $\left(\sigma_{j}^{i-1}\right)$. Define $\tau_{j}^{i-1}=f_{i-1}\left(\sigma_{j}^{i-1}\right)$ and let $\hat{\tau}_{j}^{i-1}$. be the barycenter of $\tau_{j}^{i-1}$. For each $j$, there is a simplex $D_{j}^{n-i}$ of $\left(K^{n-i}\right)^{\sim}$ such that $\tau_{j}^{i-1}$ is contained in $v\left(\right.$ bdy $\left.\left(D_{j}^{n-i}\right)\right)$. Let $\left(v K^{n-i}\right)^{\approx}$ $=$ the extension of $\left(f_{i-1}\left(X_{i-1}^{*}\right)\right)^{\tilde{z}}$ to $\left(v K^{n-i}\right)^{\sim}$. Use the ordering on the $\tau_{j}^{i-1}$ induced by the ordering on the $\sigma_{j}^{i-1}$, and construct inductively a sequence of new vertices $a_{j}^{i-1}$ and $c_{j}^{i-1}$, and a sequence of subdivisions of $\left(v K^{n-i}\right)^{\tau}$; do this by choosing 
$a_{j}^{i-1}$ on the ray $v \hat{\tau}_{j}^{i-1}$ and in the interior of the unique simplex of the $(j-1)$ th subdivision that is contained in $v\left(\right.$ bdy $\left.\left(D_{j}^{n-i}\right)\right)$, that contains $\tau_{j}^{i-1}$ as a top-dimensional face, and whose interior intersects the line segment $v \hat{\tau}_{j}^{i-1}$. Subdivide the $(j-1)$ th subdividion by starring at $a_{j}^{i-1}$. Choose $c_{j}^{i-1}$ to be empty if $\tau_{j}^{i-1}$ is contained in $D_{j}^{n-i}$ itself. Otherwise choose $c_{j}^{i-1}$ to be on the ray extending $v \hat{\tau}_{j}^{i-1}$ and in the interior of the unique simplex, in the subdivision last described, that has $\tau_{j}^{i-1}$ as a top-dimensional face and whose interior does not intersect $v \hat{\tau}_{j}^{i-1}$. Obtain the $j$ th subdivision by starring at $c_{j}^{i-1}$. We produce the sets $a_{j}^{i-1} \tau_{j}^{i-1} c_{j}^{i-1}$ by this construction. These sets are contained in $v K^{n-i-1}$, and they have the property

$$
a_{j}^{i-1} \tau_{j}^{i-1} c_{j}^{i-1} \cap a_{w}^{i-1} \tau_{w}^{i-1} c_{w}^{i-1}=\tau_{j}^{i-1} \cap \tau_{w}^{i-1} .
$$

Similarly, construct inductively a sequence of vertices $b_{j}^{i-1}$ and a new sequence of subdivisions of the $r$ th (last) one obtained above by choosing $b_{j}^{i-1}$ in the interior of the small star of $\tau_{j}^{i-1}$ in the new $(j-1)$ th subdivision (of the aforementioned $r$ th subdivision). The modification of Zeeman's construction comes at this point. If $\tau_{j}^{i-1}$ is contained in bdy $\left(A_{s}\right)$ (for some $s=-1, \ldots, n+1$ ), then choose $b_{j}^{i-1}$ also in bdy $\left(A_{s}\right)$; this makes sense since both $\tau_{j}^{i-1}$ and $A_{s}$ are polyhedra in $I^{n}$, and does not conflict with Zeeman's choice since $f\left(I^{k} \times\right.$ bdy $\left.\left(I^{n-k}\right)\right)$ contains no singular points.

Define blisters $Z_{j}^{i-1}=a_{j}^{i-1} b_{j}^{i-1} \tau_{j}^{i-1} c_{j}^{i-1}$. Notice that

$$
Z_{j}^{i-1} \cap Z_{w}^{i-1}=\tau_{j}^{i-1} \cap \tau_{w}^{i-1} .
$$

Define $e_{i} \mid a_{j}^{i-1} \tau_{j}^{i-1} c_{j}^{i-1}$ as the simplicial homeomorphism specified by the requirements that

and

$$
e_{i}\left(\hat{\tau}_{j}^{i-1}\right)=b_{j}^{i-1}
$$

$$
e_{i} \mid \operatorname{bdy}\left(a_{j}^{i-1} \tau_{j}^{i-1} c_{j}^{i-1}\right)=\text { the identity. }
$$

This map extends to all of the set $v K^{n-i-1}$ by the identity. The extension completes the definition of the embedding $e_{i}: v K^{n-i-1} \rightarrow v K^{n-i}$. Note that $a_{j}^{i-1}, b_{j}^{i-1}$, and $c_{j}^{i-1}$ can be chosen arbitrarily close to $\hat{\tau}_{j}^{i-1}$ and consequently, $Z_{j}^{i-1}$ can be put in an arbitrarily small neighborhood of $\tau_{j}^{i-1}$ in $I^{n}$.

Define

$$
X_{i}=f_{i-1}^{-1}\left(e_{i}\left(v K^{n-i-1}\right)\right) \text { and } f_{i}=e_{i}^{-1} \circ f_{i-1} \circ \text { inc, }
$$

where inc is the inclusion of $X_{i}$ in $X_{i-1}$.

The modification mentioned above insures that (for each $i$ ) the embedding $e_{i}: v K^{n-i-1} \rightarrow v K^{n-i}$ satisfies the two inclusions

and

$$
e_{i}\left(v K^{n-i-1} \cap A_{j}\right) \subset v K^{n-i} \cap A_{j}
$$

$$
e_{i}\left(v K^{n-i-1} \cap I^{n}-A_{j}\right) \subset v K^{n-i} \cap \bar{I}^{n}-A_{j}
$$

(for all $j=-1, \ldots, n+1$ ). 
It is implicit in this construction that

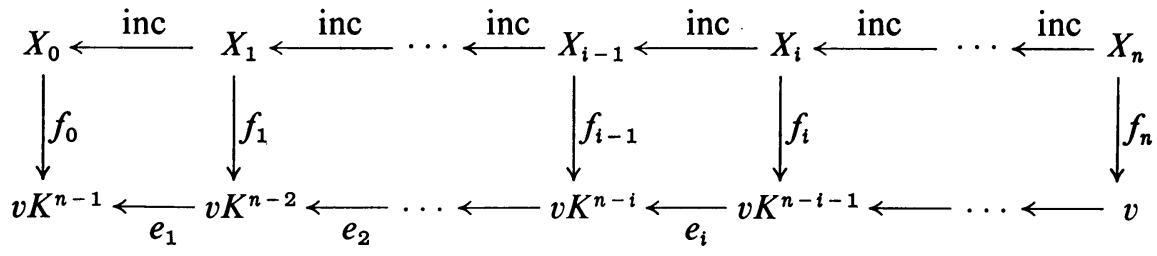

is a commutative diagram, and that $X_{j}^{*}$ is contained in $f_{j}^{-1}\left(v K^{n-j-2}\right)$. The second statement means that no point in $f_{j}^{-1}\left(v K^{n-j-1}-v K^{n-j-2}\right)$ shadows or is shadowed by any point in $f_{j}^{-1}\left(v K^{n-j-1}\right)\left(=X_{j}\right)$. A further consequence of the construction is that $X_{i-2}$, and therefore $f_{i-2}^{-1}\left(Z_{j}^{i-2}\right)$, which is contained in $X_{i-2}$, can shadow points in $X_{i-1}\left(=f_{i-1}^{-1}\left(v K^{n-i}\right)\right)$ only if they also lie in $f_{i-1}^{-1}\left(v K^{n-i-1}\right)$. Let $Z^{i}=\bigcup_{j} Z_{j}^{i}$. This completes Zeeman's construction and the list of its consequences that we use in the rest of the proof of Lemma 3.

Define a new sequence $W_{i}$ of subpolyhedra of $I_{2}^{k} \times I^{q-k}$ by $W_{0}=X_{0}$ and

$$
W_{i}=X_{i} \bigcup_{j=0}^{i-1}\left(f_{j}^{-1}\left(\left(v K^{n-j-1} \cap A_{j}\right) \cup \zeta^{j}\right)\right)
$$

$(i=1, \ldots, n)$. The set $\zeta^{j}$ is the union of those blisters $Z_{w}^{j} \subset A_{j+1}$ for which $f_{j}^{-1}$ (int $\left(\tau_{w}^{j}\right)$ ) shadows $f_{j}^{-1}\left(\right.$ int $\left.\left(\tau_{r}^{j}\right)\right)$ implies that $Z_{r}^{j} \subset A_{j+1}$.

The remainder of the proof shows that $W_{i-1}$ sunny collapses to $W_{i}(i=1, \ldots, n)$, and that $W_{n}$ (which we call $R$ ) satisfies the requirements of the lemma. It is convenient to apply the homeomorphism $f_{0}$ to the sets $W_{i}$, and then work in $I^{n}$ $\left(=f_{0}\left(X_{0}\right)\right)$ to find a collapse, of $f_{0}\left(W_{i-1}\right)$ to $f_{0}\left(W_{i}\right)$, whose inverse image under $f_{0}$ is a sunny collapse from $W_{i-1}$ to $W_{i}$.

The commutative diagram gives the equalities:

$$
\begin{aligned}
f_{0}\left(W_{i-1}\right) & =f_{0}\left(X_{i-1}\right) \bigcup_{j=0}^{i-2} f_{0} f_{j}^{-1}\left(\left(A_{j} \cap v K^{n-j-1}\right) \cup \zeta^{j}\right) \\
& =e_{1} \cdots e_{i-1} f_{i-1}\left(X_{i-1}\right) \bigcup_{j=0}^{i-2} e_{1} \cdots e_{j}\left(\left(A_{j} \cap v K^{n-j-1}\right) \cup \zeta^{j}\right) \\
& =e_{1} \cdots e_{i-1}\left(v K^{n-1}\right) \bigcup_{j=0}^{i-2} e_{1} \cdots e_{j}\left(\left(A_{j} \cap v K^{n-j-1}\right) \cup \zeta^{j}\right) .
\end{aligned}
$$

For each principal simplex $D$ in $\left(K^{n-i}\right)^{\sim}$ that is not contained in $A_{i-1}$, the set $\overline{v D-A_{i-1}}$ is a $(n-i+1)$-dimensional cell that collapses in $v K^{n-i}$ through the $(n-i)$-face $D$. The simplicial map obtained by taking $\hat{\tau}_{j}^{i-1}$ onto $b_{j}^{i-1}$ (for all $j$ ) induces a homeomorphism of $\overline{v D-A_{i-1}}$ onto $\overline{v D-\left(A_{i-1} \cup Z^{i-1}\right)}$ that takes $D$ onto $\overline{D-Z^{i-1}}$. Thus

$$
e_{1} \circ \cdots \circ e_{i-1} \overline{\left(v D-\left(A_{i-1} \cup Z^{i-1}\right)\right)}
$$

collapses in $e_{1} \circ \cdots \circ e_{i-1}\left(v K^{n-i}\right)$ through $e_{1} \circ \cdots \circ e_{i-1} \overline{\left(D-Z^{i-1}\right)}$. 
Notice, in addition, that $e_{1} \circ \ldots \circ e_{i-1} \overline{\left(v D-\left(A_{i-1} \cup Z^{i-1}\right)\right)}$ is contained in $\overline{I^{n}-A_{i-1}}$. The set $e_{1} \circ \cdots \circ e_{i-1} \overline{\left(D-Z^{i-1}\right)}$ can intersect $A_{i-1}$ only in

$$
\text { bdy } \left.\left(e_{1} \circ \cdots \circ e_{i-1} \overline{\left(D-Z^{i-1}\right.}\right)\right) \text {. }
$$

Also

$$
f_{0}\left(W_{i-1}\right)-e_{1} \circ \cdots \circ e_{i-1}\left(v K^{n-i}\right) \subset A_{i-1}
$$

Consequently,

$$
e_{1} \circ \cdots \circ e_{i-1} \overline{\left(v D-\left(A_{i-1} \cup Z^{i-1}\right)\right)}
$$

collapses through $e_{1} \circ \cdots \circ e_{i-1} \overline{\left(D-Z^{i-1}\right)}$ in $f_{0}\left(W_{i-1}\right)$.

The points removed by this collapse are

$$
S^{1} \equiv e_{1} \circ \cdots \circ e_{i-1}\left(\operatorname{int} \overline{\left(v D-\left(A_{i-1} \cup Z^{i-1}\right)\right)} \cup \operatorname{int} \overline{\left(D-Z^{i-1}\right.}\right) \text {. }
$$

To show that the inverse image under $f_{0}$ of the collapse is sunny, it is sufficient to show that none of the points in $S^{1}$ has inverse image shadowed by a point in $W_{i-1}\left(=f_{0}^{-1} f_{0}\left(W_{i-1}\right)\right)$. Observe that

$$
W_{i-1}=f_{0}^{-1} \circ e_{1} \circ \cdots \circ e_{i-1}\left(v K^{n-i}\right) \bigcup_{j=0}^{i-2} f_{0}^{-1} \circ e_{1} \circ \cdots \circ e_{j}\left(\left(A_{j} \cap v K^{n-j-1}\right) \cup \zeta^{j}\right) .
$$

Also

$$
\begin{aligned}
f_{0}^{-1}\left(S^{1}\right) & =f_{0}^{-1}\left(e_{1} \circ \cdots \circ e_{i-1}\left(\operatorname{int} \overline{\left(v D-\left(A_{i-1} \cup Z^{i-1}\right)\right)} \cup \operatorname{int} \overline{\left(D-Z^{i-1}\right.}\right)\right. \\
& =f_{i-1}^{-1}\left(\operatorname{int} \overline{\left(v D-\left(A_{i-1} \cup Z^{i-1}\right)\right)} \cup \operatorname{int} \overline{\left(D-Z^{i-1}\right.}\right) \\
& \subset f_{i-1}^{-1}\left(v K^{n-i}-v K^{n-i-1}\right)
\end{aligned}
$$

this means $f_{0}^{-1}\left(S^{1}\right)$ is not shadowed by $f_{0}^{-1} \circ e_{1} \circ \cdots \circ e_{i-1}\left(v K^{n-i}\right)\left(=f_{i-1}^{-1}\left(v K^{n-i}\right)\right)$. In addition,

$$
\begin{aligned}
& \bigcup_{j=0}^{i-2} f_{0}^{-1} \circ e_{1} \circ \cdots \circ e_{j}\left(\left(A_{j} \cap v K^{n-j-1}\right) \cup \zeta^{j}\right) \\
& \qquad f_{0}^{-1}\left(A_{i-2}\right) \cup f_{0}^{-1} \circ e_{1} \circ \cdots \circ e_{i-2}\left(\zeta^{i-2}\right) .
\end{aligned}
$$

The set $f_{0}^{-1}\left(A_{i-2}\right)\left(=f\left(A_{i-2}\right)\right)$ is within $\delta$ of $A_{i-2} \subset I_{2}^{k} \times I^{q-k}$ and therefore cannot shadow $f \overline{\left(I^{n}-A_{i-1}\right)}$. The inverse image of $S^{1}$ is contained in the last set.

Finally, it is one of the previously mentioned consequences of Zeeman's construction that $f_{0}^{-1} \circ e_{1} \circ \cdots \circ e_{i-2}\left(\zeta^{i-2}\right)\left(=f_{i-2}^{-1}\left(\zeta^{i-2}\right)\right)$ does not shadow

$$
f_{i-1}^{-1}\left(v K^{n-i}-v K^{n-i-1}\right) \text {. }
$$

This last set contains $f_{0}^{-1}\left(S^{1}\right)$.

If the above procedure is carried out for all principal simplexes $D$ in $K^{n-i}$ that are not contained in $A_{i-1}$, then a collapse is obtained, from

$$
e_{1} \circ \ldots \circ e_{i-1}\left(v K^{n-i}\right) \bigcup_{j=0}^{i-2} e_{1} \circ \cdots \circ e_{j}\left(\left(A_{j} \cap v K^{n-j-1}\right) \cup \zeta^{j}\right)
$$


to

$$
\begin{aligned}
& e_{1} \circ \cdots \circ e_{i-1}\left(e_{i} v K^{n-i-1}\right) \cup e_{1} \circ \cdots \circ e_{i-1}\left(v K^{n-i} \cap A_{i-1}\right) \\
& \cup e_{1} \circ \cdots \circ e_{i-1}\left(Z^{i-1}\right) \bigcup_{j=0}^{i-2} e_{1} \circ \cdots \circ e_{j}\left(\left(A_{j} \cap v K^{n-i-1}\right) \cup \zeta^{j}\right) \equiv S^{2},
\end{aligned}
$$

whose inverse image under $f_{0}$ is sunny.

Each $Z_{j}^{i-1}$ not in $\zeta^{i-1}$ is in the $3 \delta$-neighborhood of $\overline{\left(I^{n}-A_{i}\right)}$, since if it is not already in $\overline{\left(I^{n}-A_{i}\right)}$, then $f_{i-1}^{-1}\left(\right.$ int $\left.\left(\tau_{j}^{i-1}\right)\right)$ must shadow $f_{i-1}^{-1}\left(\right.$ int $\left.\left(\tau_{w}^{i-1}\right)\right)$ for some $\left.\tau_{w}^{i-1} \subset \overline{\left(I^{n}-A_{i}\right.}\right)$. Consequently, the cell $e_{1} \circ \ldots \circ e_{i-1}\left(Z_{j}^{i-1}\right)$ does not intersect $A_{i-1}$ and has its top-dimensional face $e_{1} \circ \ldots \circ e_{i-1}\left(a_{j}^{i-1} \tau_{j}^{i-1} c_{j}^{i-1}\right)$ free in $S^{2}$; this means that the cell collapses to (bdy $\left.\left(a_{j}^{i-1} \tau_{j}^{i-1} c_{j}^{i-1}\right)\right) b_{j}^{i-1}$.

The inverse image under $f_{0}$ of the points removed in such a collapse lies in $f_{0}^{-1} \circ e_{1} \circ \cdots \circ e_{i-1}\left(Z_{j}^{i-1}\right)\left(=f_{i-1}^{-1}\left(Z_{j}^{i-1}\right)\right)$. The union

$$
f_{0}^{-1}\left(e_{1} \circ \cdots \circ e_{i-1}\left(v K^{n-i} \cap A_{i-1}\right) \bigcup_{j=0}^{i-2} e_{1} \circ \cdots \circ e_{j}\left(\left(A_{j} \cap v K^{n-j-1}\right) \cup \zeta^{j}\right)\right)
$$

lies in the $\delta$-neighborhood of $A_{i-1} \subset I_{2}^{k} \times I^{q-k}$, and thus cannot shadow points in $f_{i-1}^{-1}\left(Z_{j}^{i-1}\right)$ for any $Z_{j}^{i-1}$ not in $\zeta^{i-1}$. The set

$$
f_{0}^{-1} \circ e_{1} \circ \cdots \circ e_{i}\left(v K^{n-i-1}\right)=X_{i},
$$

and hence does not shadow points in

$$
f_{0}^{-1} \circ e_{1} \circ \cdots \circ e_{i-1}\left(\operatorname{int}\left(Z_{j}^{i-1}\right) \cup \operatorname{int}\left(a_{j}^{i-1} \tau_{j}^{i-1} c_{j}^{i-1}\right)\right)
$$

(for any $j$ ). We know $f_{i-1}^{-1}\left(Z_{w}^{i-1}\right)$ shadows points in

$$
f_{i-1}^{-1}\left(\operatorname{int}\left(Z_{j}^{i-1}\right) \cup \operatorname{int}\left(a_{j}^{i-1} \tau_{j}^{i-1} c_{j}^{i-1}\right)\right) \equiv S^{3}
$$

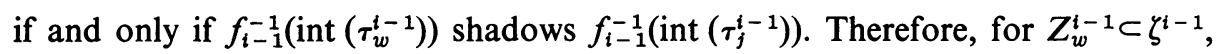
the set $f_{i-1}^{-1}\left(Z^{i-1}\right)$ shadows $S^{3}$ only if $Z_{j}^{i-1}$ is also in $\zeta^{i-1}$. For the $Z_{j}^{i-1}$ that are not in $\zeta^{i-1}$, the cells $e_{1} \circ \cdots \circ e_{i-1}\left(Z_{j}^{i-1}\right)$ collapse (in order of increasing $j$ ) to

$$
e_{1} \circ \cdots \circ e_{i-1}\left(\left(\operatorname{bdy}\left(a_{j}^{i-1} \tau_{j}^{i-1} c_{j}^{i-1}\right)\right) b_{j}^{i-1}\right) \text {. }
$$

The inverse image under $f_{0}$ of these collapses is sunny for precisely the same reason as in Zeeman's proof. Note that $f_{0}^{-1} \circ e_{1} \circ \ldots \circ e_{i-1}\left(\zeta^{i-1}\right)$ is still undisturbed.

The set $f\left(I^{n}-A_{0}\right)$ is contained in the $\delta$-neighborhood of $\left(I^{n}-A_{0}\right)$ in $I_{2}^{k} \times I^{q-k}$, and therefore does not intersect $A\left(=A_{-1} \times I^{q-n}\right)$. Thus

$$
f\left(I^{n}\right) \cap A \subset f\left(A_{0}\right)=f_{0}^{-1}\left(A_{0}\right) \subset \bigcup_{j=0}^{n-1} f_{j}^{-1}\left(\left(A_{j} \cap v K^{n-j-1}\right) \cup \zeta^{j}\right)=R .
$$

Also,

$$
f_{j}^{-1}\left(\left(A_{j} \cap v K^{n-j-1}\right) \cup \zeta^{j}\right) \subset f_{j}^{-1}\left(A_{j+1} \cap v K^{n-j-1}\right) \subset f_{0}^{-1}\left(A_{j+1}\right)
$$

(for all $j \leqq(n-1)$ ). This means $R \subset f_{0}^{-1}\left(A_{n}\right)$. But $f_{0}^{-1}\left(A_{n}\right)\left(=f\left(A_{n}\right)\right)$ is contained in the $\delta$-neighborhood of $A_{n}$ in $I_{2}^{k} \times I^{q-k}$, and consequently cannot intersect $\left(I_{2}^{k} \times I^{q-k}\right)-\left(A_{n+1} \times I^{q-n}\right)$. The last set equals $\left(I_{2}^{k} \times I^{q-k}\right)-B$. 
Finally, it is necessary to verify that $\hat{K}$ sunny collapses to the point $f(v)$. We do this in stages by showing that $\hat{K} \cap X_{i-1}$ sunny collapses to $\hat{K} \cap X_{i}$ (for each $i$ ). Again it is convenient to collapse $f_{0}\left(\hat{R} \cap X_{i-1}\right)$ to $f_{0}\left(R \cap X_{i}\right)$ and check that the inverse image of this collapse under $f_{0}$ is sunny.

We have the equalities:

$$
\hat{K} \cap X_{i-1}=\bigcup_{j=i-1}^{n-1} f_{j}^{-1}\left(\left(A_{j} \cap v K^{n-j-1}\right) \cup \zeta^{j}\right)
$$

and

$$
f_{0}\left(R \cap X_{i-1}\right)=\bigcup_{j=i-1}^{n-1} e_{1} \circ \cdots \circ e_{j}\left(\left(A_{j} \cap v K^{n-j-1}\right) \cup \zeta^{j}\right) .
$$

For each principal simplex $D$ in $\left(K^{n-i}\right)^{\sim}$, the cell $v D \cap A_{i-1}$ collapses through $v D \cap$ bdy $\left(A_{i-1}\right)$ in $v K^{n-i} \cap A_{i-1}$. As before, the simplicial map that takes $\hat{\tau}_{j}^{i-1}$ onto $b_{j}^{i-1}$ (for all $j$ ) induces a homeomorphism of $v D \cap A_{i-1}$ onto

$$
\overline{\left(v D \cap A_{i-1}\right)-\zeta^{i-1}}
$$

that takes $v D \cap$ bdy $\left(A_{i-1}\right)$ onto

$$
\overline{\left(v D \cap \text { bdy }\left(A_{i-1}\right)\right)-\zeta^{i-1}} \text {. }
$$

Thus $\overline{\left(v D \cap A_{i-1}\right)-\zeta^{i-1}}$ collapses through the last displayed set in $v K^{n-i} \cap A_{i-1}$. Since

$$
\begin{aligned}
\zeta^{i-1} \cap \overline{\left(\left(v D \cap A_{i-1}\right)-\zeta^{i-1}\right)} \\
\quad \subset \operatorname{bdy} \overline{\left(\left(v D \cap A_{i-1}\right)-\zeta^{i-1}\right)}-\operatorname{int} \overline{\left(\left(v D \cap \operatorname{bdy}\left(A_{i-1}\right)\right)-\zeta^{i-1}\right)},
\end{aligned}
$$

the cell $\overline{\left(v D \cap A_{i-1}\right)-\zeta^{i-1}}$ collapses through $\overline{\left(v D \cap \text { bdy }\left(A_{i-1}\right)\right)-\zeta^{i-1}}$ in

Thus

$$
\left(v K^{n-i} \cap A_{i-1}\right) \cup \zeta^{i-1} \text {. }
$$

$$
e_{1} \circ \cdots \circ e_{i-1} \overline{\left(\left(v D \cap A_{i-1}\right)-\zeta^{i-1}\right)}
$$

collapses through $e_{1} \circ \ldots \circ e_{i-1} \overline{\left.\left(v D \cap \operatorname{bdy}\left(A_{i-1}\right)\right)-\zeta^{i-1}\right)}$ in

$$
e_{1} \circ \cdots \circ e_{i-1}\left(\left(v K^{n-i} \cap A_{i-1}\right) \cup \zeta^{i-1}\right) \text {. }
$$

Furthermore,

$$
\begin{aligned}
e_{1} \circ \cdots \circ & e_{i-1} \overline{\left(\left(v D \cap A_{i-1}\right)-\zeta^{i-1}\right)} \cap\left(\bigcup_{j=1}^{n-1} e_{1} \circ \cdots \circ e_{j}\left(\left(A_{j} \cap v K^{n-j-1}\right) \cup \zeta^{j}\right)\right) \\
& \left.\subset e_{1} \circ \cdots \circ e_{i-1} \overline{\left(\left(v D \cap A_{i-1}\right)-\zeta^{i-1}\right.}\right) \cap e_{1} \circ \cdots \circ e_{i}\left(v K^{n-i-1}\right) \\
& \left.=e_{1} \circ \cdots \circ e_{i-1}\left(\overline{\left(v D \cap A_{i-1}\right)-\zeta^{i-1}}\right) \cap e_{i}\left(v K^{n-i-1}\right)\right) \\
& \subset e_{1} \circ \cdots \circ e_{i-1}\left(\operatorname{bdy} \overline{\left(\left(v D \cap A_{i-1}\right)-\zeta^{i-1}\right.}\right)-\operatorname{int} \overline{\left.\left(\left(v D \cap \operatorname{bdy}\left(A_{i-1}\right)\right)-\zeta^{i-1}\right)\right)} .
\end{aligned}
$$

Thus, $e_{1} \circ \ldots \circ e_{i-1} \overline{\left.\left(v D \cap A_{i-1}\right)-\zeta^{i-1}\right)}$ collapses through

$$
e_{1} \circ \cdots \circ e_{i-1} \overline{\left.\left(v D \cap \operatorname{bdy}\left(A_{i-1}\right)\right)-\zeta^{i-1}\right)}
$$


in $f_{0}\left(R \cap X_{i-1}\right)$. The set removed by the collapse is

$$
\left.S^{4} \equiv e_{1} \circ \cdots \circ e_{i-1}\left(\text { int } \overline{\left(\left(v D \cap A_{i-1}\right)-\zeta^{i-1}\right)} \cup \operatorname{int} \overline{\left(\left(v D \cap \text { bdy }\left(A_{i-1}\right)\right)-\zeta^{i-1}\right.}\right)\right) \text {. }
$$

The set $S^{4}$ is contained in $e_{1} \circ \cdots \circ e_{i-1}\left(v K^{n-i}-v K^{n-i-1}\right)$, and thus

$$
f_{0}^{-1}\left(S^{4}\right) \subset f_{0}^{-1} e_{1} \circ \cdots \circ e_{i-1}\left(v K^{n-i}-v K^{n-i-1}\right)=f_{i-1}^{-1}\left(v K^{n-i}-v K^{n-i-1}\right) .
$$

It is one of the consequences of Zeeman's construction that the last set is not shadowed by $X_{i-1}$. Therefore, the inverse image under $f_{0}$ of the collapse is sunny.

Carry out this procedure for all principal simplexes $D$ in $\left(K^{n-i}\right)^{\sim}$, and thereby obtain a collapse, from $f_{0}\left(R \cap X_{i-1}\right)$ to

$$
e_{1} \circ \cdots \circ e_{i-1}\left(\zeta^{i-1}\right) \bigcup_{j=1}^{n-1} e_{1} \circ \cdots \circ e_{j}\left(\left(A_{j} \cap v K^{n-j-1}\right) \cup \zeta^{j}\right),
$$

whose inverse image under $f_{0}$ is sunny.

Since $\bigcup_{j=i}^{n-1} e_{1} \circ \cdots \circ e_{j}\left(\left(A_{j} \cap v K^{n-j-1}\right) \cup \zeta^{j}\right)$ is contained in $e_{1} \circ \cdots \circ e_{i}\left(v K^{n-i-1}\right)$, for each blister $Z_{j}^{i-1}$ in the union $\zeta^{i-1}$, the cells $e_{1} \circ \ldots \circ e_{i-1}\left(Z_{j}^{i-1}\right)$ collapse to

$$
e_{1} \circ \cdots \circ e_{i-1}\left(e_{i}\left(a_{j}^{i-1} \tau_{j}^{i-1} c_{j}^{i-1}\right)\right)
$$

in order of increasing $j$. The inverse image under $f_{0}$ of these collapses is sunny. Take this collapse together with the collapse of the previous paragraph, and get a sunny collapse from $\hat{K} \cap X_{i-1}$ to $\hat{K} \cap X_{i}$. This completes the proof of Lemma 3.

REMARK 4. (Refer to the proof of Lemma 3 for notation.) For each principal simplex $D$ in bdy $\left(I^{n}\right)$, the image of $\overline{v D-A_{0}}$ under the collapse from $I^{n}$ to $f_{0}(\hat{K})$ is contained in the $\delta$-neighborhood of $v D \cap \overline{A_{n}-A_{0}}$.

Proof. Let $D_{i-1}$ be a principal simplex in $\left(K^{n-i}\right)^{\sim}$. During the first part of the $i$ th stage of the collapse, $e_{1} \circ \cdots \circ e_{i-1}\left(\overline{v D_{i-1}-A_{0}}\right)$ collapses to

$$
\left.e_{1} \circ \cdots \circ e_{i-1}\left(\overline{v D_{i-1}-A_{0}}-\left(\operatorname{int} \overline{\left(v D_{i-1}-\left(A_{i-1} \cup Z^{i-1}\right)\right.}\right) \cup \operatorname{int} \overline{\left(D_{i-1}-Z^{i-1}\right)}\right)\right) \text {. }
$$

This equals

$$
\begin{aligned}
e_{1} \circ \ldots \circ e_{i-1}\left(\left(v D_{i-1} \cap \overline{\left(A_{i-1}-A_{0}\right)}\right)\right. \\
\left.\cup\left(Z^{i-1} \cap \overline{\left(v D_{i-1}-A_{0}\right)}\right) \cup \overline{\left(v \cdot \mathrm{bdy}\left(D_{i-1}\right)-A_{0}\right)}\right),
\end{aligned}
$$

which, in turn, is contained in

$$
e_{1} \circ \cdots \circ e_{i-1}\left(\left(v D_{i-1} \cap \overline{\left(A_{i-1}-A_{0}\right)}\right) \cup \tilde{Z}^{i-1} \cup \overline{\left(v \cdot \operatorname{bdy}\left(D_{i-1}\right)-A_{0}\right)}\right) \equiv S^{5} .
$$

The set $\tilde{Z}^{i-1}$ is the union of all $Z_{j}^{i-1}$ which intersect $\overline{v D_{i-1}-A_{0}}$. The image of $S^{5}$ under the second part of the $i$ th stage of the collapse is

$$
e_{1} \circ \ldots \circ e_{i-1}\left(\left(\left(v D_{i-1} \cap \overline{A_{i-1}-A_{0}}\right) \cup \tilde{\zeta}^{i-1}\right) \cup e_{i}\left(\overline{\left.v \cdot \operatorname{bdy}\left(D_{i-1}\right)-A_{0}\right)}\right) \equiv S^{6},\right.
$$

where $\tilde{\zeta}^{i-1}=\zeta^{i-1} \cap \tilde{Z}^{i-1}$. Then

$$
S^{6}=e_{1} \circ \cdots \circ e_{i-1}\left(\left(v D_{i-1} \cap \overline{A_{i-1}-A_{0}}\right) \cup \tilde{\zeta}^{i-1}\right) \bigcup_{D_{i}} e_{1} \circ \cdots \circ e_{i} \overline{\left(v D_{i}-A_{0}\right)},
$$

where the union is over all principal simplexes $D_{i}$ in bdy $\left(D_{i-1}\right)$. Notice that 
nothing in $A_{i-1}$ is moved during the $i$ th stage of the collapse, and that the first term in $S^{6}$ is contained in $A_{i}$. Thus the image of $\overline{v D-A_{0}}$ under the entire collapse is contained in

$$
\bigcup_{i, D_{i-1}} e_{1} \circ \cdots \circ e_{i-1}\left(\left(v D_{i-1} \cap \overline{\left(A_{i-1}-A_{0}\right)}\right) \cup \tilde{\zeta}^{i-1}\right) \equiv S^{7},
$$

where the union is over all principal simplexes $D_{i-1}$ in $\left(K^{n-1}\right)^{\sim}$ that also lie in $|D|$, and over all $i$.

We noted when we defined the blisters that $a_{j}^{i-1}, b_{j}^{i-1}$, and $c_{j}^{i-1}$ can all be chosen arbitrarily close to $\hat{\tau}_{j}^{i-1}$, and consequently $Z_{j}^{i-1}$ can be put in an arbitrarily small neighborhood of $\tau_{j}^{i-1}$ in $I^{n}$. Also, by its construction, the map $e_{i}$ moves points no more than

$$
\max _{j}\left\{\text { distance from } \hat{\tau}_{j}^{i-1} \text { to } b_{j}^{i-1}\right\} \text {. }
$$

Thus we may choose $e_{i}$ arbitrarily close to the identity. The proof of Lemma 3 makes no restriction on how small the distances from $a_{j}^{i-1}, b_{j}^{i-1}$, and $c_{j}^{i-1}$ to $\hat{\tau}_{j}^{i-1}$ can be, and we now assume that the choice is close enough to insure that the set $S^{7}$ is contained in the $\delta$-neighborhood of $v D \cap \overline{A_{n}-A_{0}}$. This completes the proof of Remark 4.

If $k=0$, let $C^{1}=\overline{I^{q}-I_{1-\mu}^{q}}$ (for some $0<\mu<\delta$ ), and if $k>0$, let

$$
C^{1}=I_{2}^{k} \times \overline{I^{q-k}-I_{1 / 2}^{q-k}} .
$$

LEMMA 5. If the embedding $f$ in Lemma 3 satisfies the additional condition that $f \mid C^{1} \cap I^{n}$ is the identity, then there is a set $K$ which satisfies all the conclusions of Lemma 3 and also satisfies

$$
K \cap \operatorname{bdy}\left(I_{2}^{k} \times I^{q-k}\right)=K \cap \operatorname{bdy}(B)=B \cap I^{n} \cap \operatorname{bdy}\left(I_{2}^{k} \times I^{q-k}\right) .
$$

Proof. If $k=0$, let $C^{2}=\overline{I^{q}-I_{1-\kappa}^{q}}$ where $0<\kappa<\mu$ and $\kappa$ is sufficiently small that $f\left(C^{2} \cap I^{n}\right)=C^{2} \cap I^{n}=f\left(I^{n}\right) \cap C^{2} \equiv S^{8}$. Let $C^{3}=\overline{I^{q}-I_{1-\kappa / 2}^{q}}$. Subdivide $I^{n}$ so that the triangulation is conical from the origin and collapse $I^{n}$ to $\left(B \cap I^{n}\right) \cup \overline{I^{n}-C^{2}}$ radially. The diameter of this collapse is less than $(n+2) \delta$, since the mesh of the triangulation is less than $\delta$, and since the radial distance from $\overline{I^{n}-C^{2}}$ to bdy $\left(I^{n}\right)$ is greatest along the ray from the origin to $(1, \ldots, 1)$ (where it is less than $\sqrt{ } n \delta$ $\leqq n \cdot \delta)$. Now collapse $\left(B \cap I^{n}\right) \cup \overline{I^{n}-C^{2}}$ cylindrically in the direction of the first coordinate to

$$
\left.\left(B \cap I^{n} \cap \operatorname{bdy}\left(I_{2}^{k} \times I^{q-k}\right)\right) \cup\left(A \cap I^{n}\right) \cup \overline{\left(I^{n}-C^{2}\right.}\right) \equiv S^{9} .
$$

The distance from $A$ to $B$ along $I$ is $(n+2) \cdot 4 \delta$, so that the diameter of the collapse is less than $(n+2) \cdot 4 \delta+2 \delta$.

If $k>0$, set $C^{2}=I_{2}^{k} \times \overline{\left(I^{q-k}-I_{3 / 4}^{q}\right)}$ and $C^{3}=I_{2}^{k} \times \overline{\left(I^{q-k}-I_{7 / 8}^{q}\right)}$. $C^{2}$ formally satisfies the equalities $S^{8}$. For each $r$-simplex $D_{r}$ in bdy $\left(I^{k}\right) \times I^{n-k}$, the projection

$$
\pi: I^{n} \rightarrow\{0\} \times I^{n-k}
$$


( $\{0\}$ is the origin in $\left.I^{k}\right)$ defines an $(r+1)$-cell $\pi^{-1}\left(\pi\left(D_{r}\right)\right)$ that contains $D_{r}$ as an $r$-cell in its boundary. Call geometric collapses along such cells $c$-radial. In particular, there is a c-radial collapse of $I^{n}$ to $S^{9}$ (remember that the definition of $S^{9}$ depends on the value of $k$ ). The diameter of the collapse is less than the radial distance from bdy $\left(I^{k}\right)$ to $A$, plus twice the mesh of the triangulation on $I^{n}$. This sum is less than $((k \cdot(n+2) \cdot 4 \delta)+2 \delta)$.

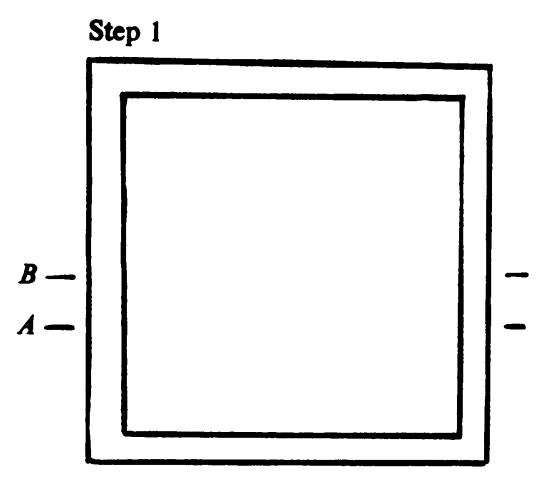

Step 2
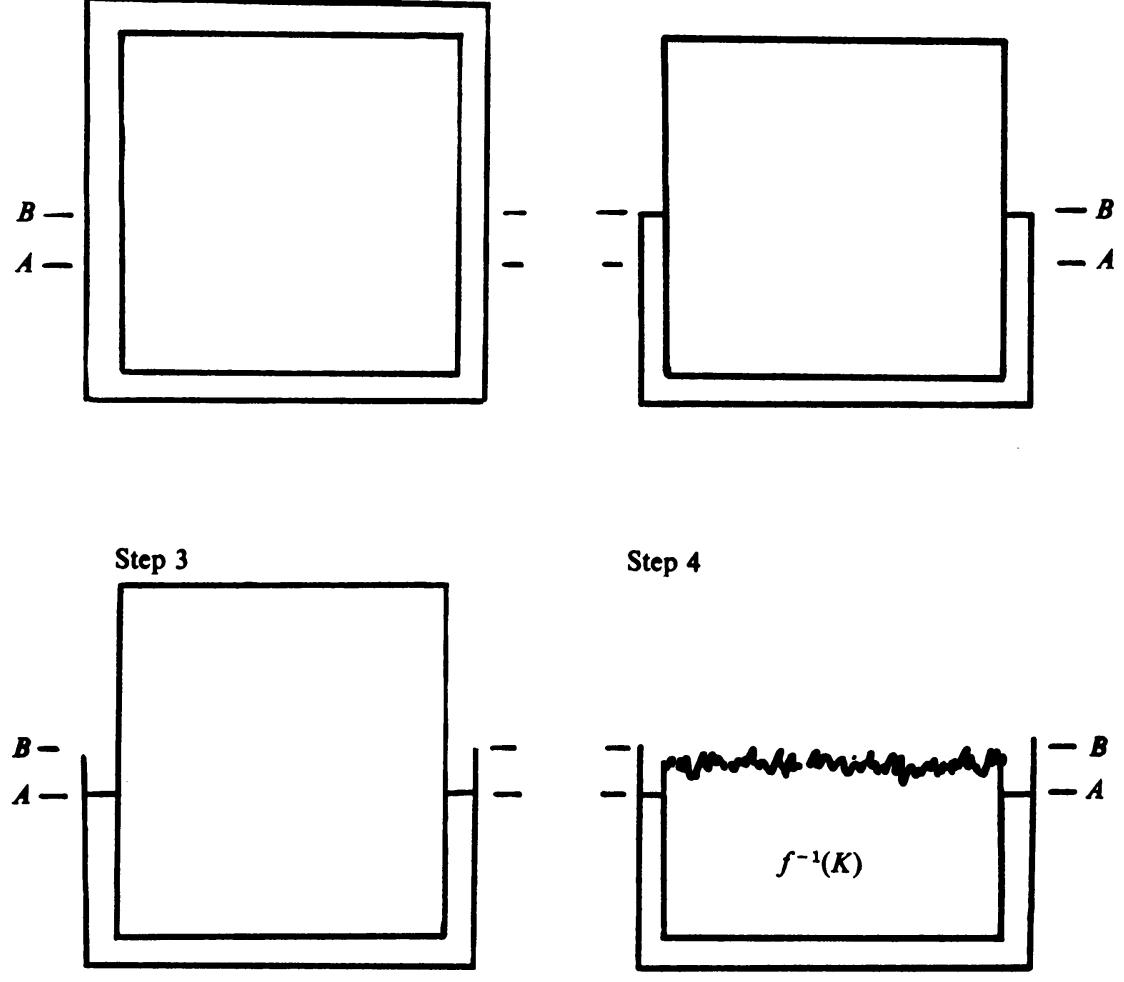

Step 4

Step 5

Step 6

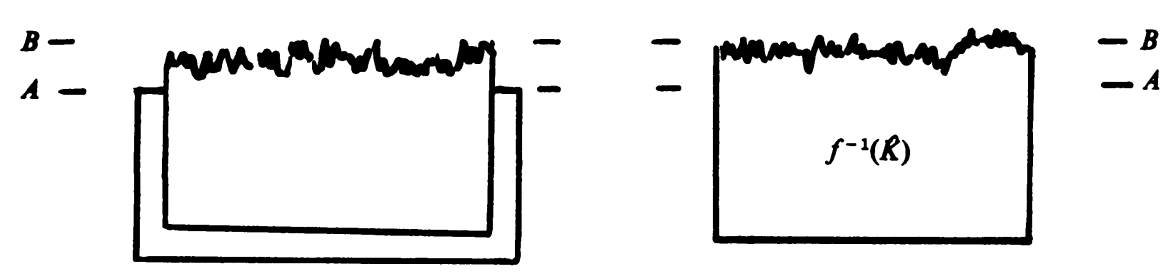

Figure 4. Lemma $5(k=0)$. 
Thus $I^{n}$ collapses to $S^{9}$. The collapse removes only points in $S^{8}\left(=C^{2} \cap I^{n}\right)$. The two other interpretations of $S^{8}$ imply that this collapse induces the identical collapse on $f\left(I^{n}\right)$, and that the latter collapse is sunny in $I_{2}^{k} \times I^{q-k}$.

Now apply Lemma 3 to

$$
f \mid \overline{I^{n}-C^{2}}: \overline{I^{n}-C^{2}} \rightarrow \overline{\left(I_{2}^{k} \times I^{q-k}\right)-C^{2}},
$$

where $A, B$, and $A_{j}$ are replaced by their intersections with $\overline{\left(I_{2}^{k} \times I^{q-k}\right)-C^{2}}$, to get a sunny collapse from $f\left(\overline{\left.I^{n}-C^{2}\right)}\right.$ to $R$. Since $f$ is within $\delta$ of the identity, Remark 4 shows that the diameter of this collapse is less than the diameter of the largest $\left(v D-A_{0}\right)$, plus $\delta$, plus $2 \delta$. The diameter of $\left(v D-A_{0}\right)$ is less than $\delta$, plus the maximum distance from $A$ to bdy $\left(I^{n}\right)$ measured along the ray from $v$. If $k>0$, Pythagoras' Theorem shows that this diameter is less than $(8 n(n+2) \delta+\delta)$; therefore the collapse from $f\left(\overline{\left.I^{n}-C^{2}\right)}\right.$ to $R$ has diameter less than $(8 n(n+2) \delta+4 \delta)$. In addition, this collapse is a collapse in $f\left(S^{9}\right)$ that is sunny in $I_{2}^{k} \times I^{q-k}$, since no point in $f\left(S^{9} \cap C^{2}\right)$ shadows any point in $f \overline{\left(I^{n}-C^{2}\right)}$. Define $K$ as the image of $f\left(S^{9}\right)$ under this collapse. Then $f\left(I^{n}\right)$ sunny collapses to $K$. The complex $K$ collapses to $\left(A \cap I^{n}\right) \cup R$,

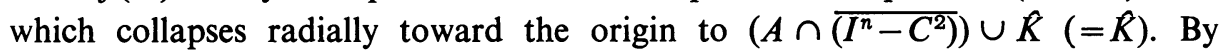
Lemma 3, $R$ sunny collapses in $\overline{\left(I_{2}^{k} \times I^{q-k}\right)-C^{2}}$ to $f(v)$. Thus $K$ collapses to $f(v)$. Again the equivalent forms of $S^{8}$ show that the collapse is actually sunny in $I_{2}^{k} \times I^{q-k}$. It is clear that $K \subset B$, that $K \supset f\left(I^{n}\right) \cap A$, and that

$$
K \cap \operatorname{bdy}\left(I_{2}^{k} \times I^{q-k}\right)=K \cap \text { bdy }(B)=B \cap I^{n} \cap \text { bdy }\left(I_{2}^{k} \times I^{q-k}\right) .
$$

REMARK 6 (Notation as in Lemma 5). Suppose $k=0$. Then there is a homeomorphism

$$
\psi: N\left(B \cap I^{n},\left(\left(B \cap I^{n}\right) \cup \overline{I^{n}-C^{3}}\right)^{\prime \prime}\right) \stackrel{\text { onto }}{\longrightarrow} N\left(f^{-1}(K),\left(\left(B \cap I^{n}\right) \cup \overline{I^{n}-C^{3}}\right)^{\prime \prime}\right)
$$

that fixes $N\left(\left(\operatorname{bdy}\left(I^{n}\right)\right) \cup\left(A \cap I^{n}\right), I^{n m}\right)$ and is within $\lambda(n) \cdot \delta$ of the identity. $\lambda(n)$ is a positive integer which depends only on $n$.

Proof. We may regard the collapse in Lemma $5(k=0)$ from $\left(B \cap I^{n}\right) \cup$ $\left(\overline{I^{n}-C^{2}}\right)$ to $S^{9}$ as a collapse from $B \cap I^{n}$ to

$$
\left(B \cap\left(\operatorname{bdy}\left(I^{n}\right) \cup \overline{I^{n}-C^{2}}\right)\right) \cup\left(A \cap I^{n}\right) \equiv S^{10} .
$$

We saw that the diameter of this collapse is less than $(4 \delta(n+2)+2 \delta)$. Since the mesh of the triangulation on $I^{n}$ is less than $\delta$, Proposition 1 gives a homeomorphism

$$
\psi^{\mathrm{I}}: N\left(B \cap I^{n},\left(\left(B \cap I^{n}\right) \cup \overline{I^{n}-C^{3}}\right)^{\prime \prime}\right) \stackrel{\text { onto }}{\longrightarrow} N\left(S^{10},\left(\left(B \cap I^{n}\right) \cup \overline{I^{n}-C^{3}}\right)^{\prime \prime}\right)
$$

that is within $(4 \delta(n+3))$ of the identity. There is a radial collapse toward $v$ of $\overline{I^{n}-C^{2}}$ to $\overline{I^{n}-C^{2}} \cap B$. Let

$$
\psi^{\mathrm{II}}: N\left(\overline{I^{n}-C^{2}}, I^{n \prime \prime}\right) \stackrel{\text { onto }}{\longrightarrow} N\left(\overline{I^{n}-C^{2}} \cap B, I^{n \prime \prime}\right)
$$

be the corresponding homeomorphism given by Proposition 1. If $D_{1}$ and $D_{2}$ are 
First Collapse

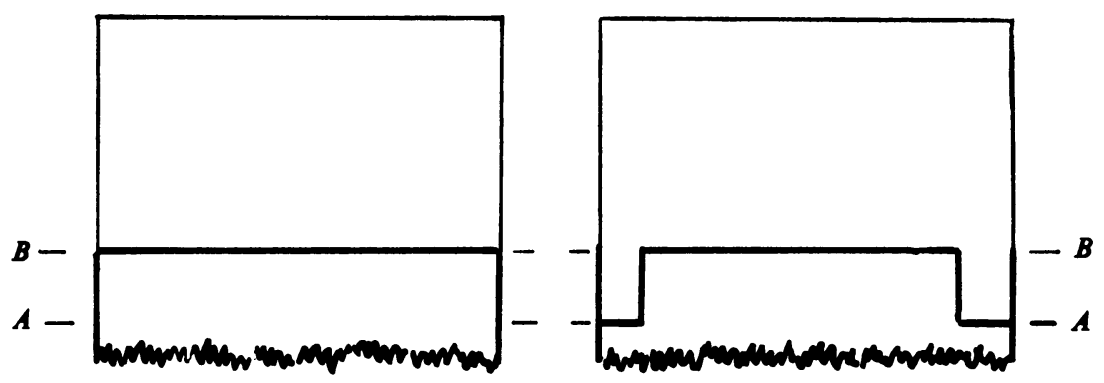

Second Collapse (radially toward $v$ )

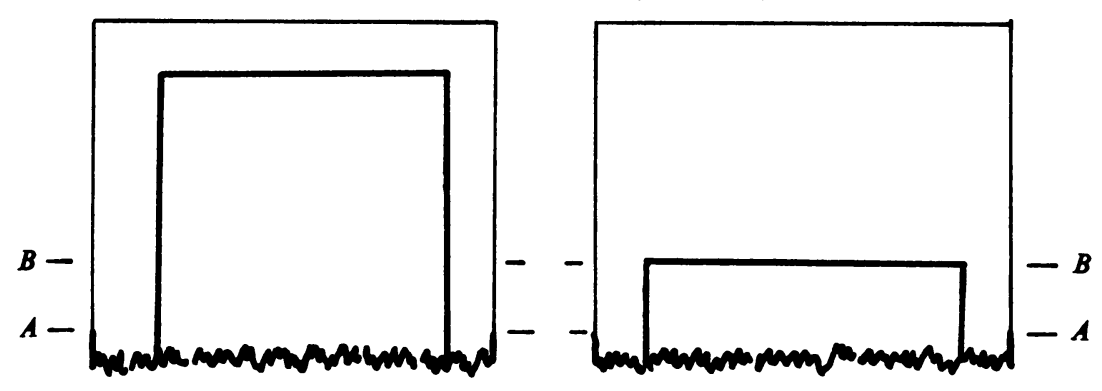

Third Collapse (by Lemma 3)

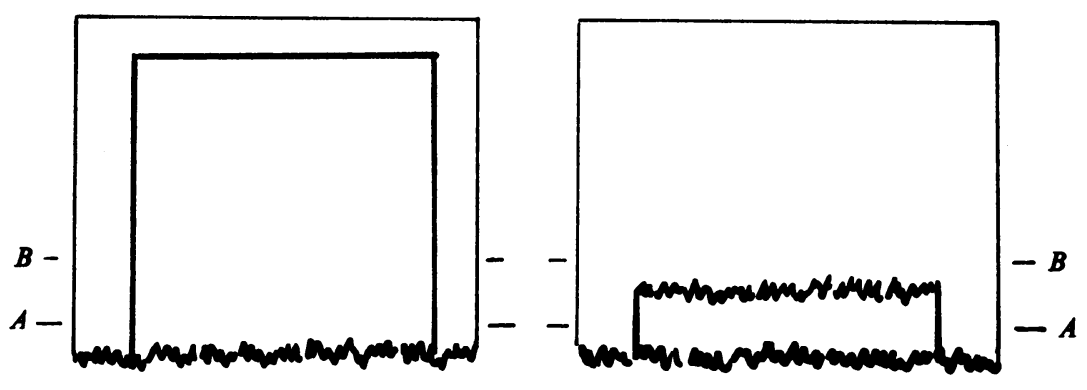

Figure 5. Remark 6.

disjoint principal simplexes in bdy $\overline{\left(I^{n}-C^{2}\right)}$, then the sets $N\left(v D_{i} \cap \overline{B-A}, I^{n \prime \prime}\right)$ are disjoint $(i=1,2)$. Since for each $i$,

$$
\psi^{\mathrm{II}}\left(N\left(\overline{v D_{i}-A}, I^{n \prime \prime}\right)\right) \subset N\left(v D_{i} \cap \overline{B-A}, I^{n \prime \prime}\right),
$$


the sets $\psi^{\mathrm{II}}\left(N\left(\overline{v D_{i}-A}, I^{n \prime}\right)\right)$ are disjoint $(i=1,2)$. Thus

$$
\begin{aligned}
\left(\psi^{\mathrm{II}}\right)^{-1}\left(N\left(v D \cap \overline{B-A}, I^{n^{\prime \prime}}\right)\right) & \subset N\left(\overline{\left(v \cdot N\left(D, \text { bdy } \overline{\left(I^{n}-C^{2}\right)}\right)-A\right.}, I^{n \prime \prime}\right) \\
& =\bigcup_{D} N \overline{\left(\overline{v D-A}, I^{n \prime \prime}\right),}
\end{aligned}
$$

where the union is over all principal simplexes in $N\left(D\right.$, bdy $\overline{\left.\left(I^{n}-C^{2}\right)\right)}$.

Let $\psi^{\mathrm{III}}$ be the homeomorphism that Proposition 1 associates with the collapse of $\overline{I^{n}-C^{2}}$ to $R$. Remark 4 shows that

$$
\psi^{\mathrm{III}}\left(N\left(v \hat{D}-A, I^{n \prime \prime}\right)\right)
$$

is contained in $N\left(\delta\right.$-neighborhood of $\left.v \hat{D} \cap \overline{B-A}, I^{n \prime \prime}\right)$. This is, in turn, contained in the $2 \delta$-neighborhood of $v \hat{D} \cap \overline{B-A}$. Thus

$$
\begin{aligned}
\psi^{\mathrm{III}} \circ\left(\psi^{\mathrm{II}}\right)^{-1}\left(N\left(v D \cap \overline{B-A}, I^{n \prime}\right)\right) & \subset \bigcup \psi^{\mathrm{III}}\left(N\left(\overline{v \overline{D-A}}, I^{n \prime \prime}\right)\right) \\
& \subset \bigcup(2 \delta \text {-neighborhood of } v D \cap \overline{B-A}) \\
& \subset(3 \delta \text {-neighborhood of } v D \cap \overline{B-A}),
\end{aligned}
$$

where the unions are over all principal simplexes $\hat{D}$ in $N\left(D\right.$, bdy $\left.\left(\overline{I^{n}-C^{2}}\right)\right)$. Consequently,

$$
\psi^{\mathrm{III}} \circ\left(\psi^{\mathrm{II}}\right)^{-1} \mid N\left(v D \cap \overline{B-A}, I^{n \prime \prime}\right)
$$

is within $(6 \delta+\operatorname{diameter}(v D \cap \overline{B-A}))$ of the identity. Pythagoras' Theorem shows that $(7 \delta+(1+4 n) \cdot 4 \delta \cdot(n+2))$ is an upper bound for this distance.

Since each of the three collapses fixes bdy $\left(I^{n}\right) \cup\left(A \cap I^{n}\right)$, Proposition 1 insures that $\psi^{\mathrm{I}}, \psi^{\mathrm{II}}$, and $\psi^{\mathrm{II}}$ each fix

$$
S^{11} \equiv N\left(\operatorname{bdy}\left(I^{n}\right) \cup\left(A \cap I^{n}\right), I^{n m}\right) .
$$

In particular then, $\psi^{\mathrm{III}} \circ\left(\psi^{\mathrm{II}}\right)^{-1}$ is within $(7 \delta+(1+4 n) 4 \delta(n+2))$ of the identity everywhere. Finally, $\psi \equiv \psi^{\mathrm{III}} \circ\left(\psi^{\mathrm{II}}\right)^{-1} \circ \psi^{\mathrm{I}}$ fixes $S^{11}$ and is within

$$
(7 \delta+(1+4 n) 4 \delta(n+2)+(n+3) 4 \delta) \equiv \lambda(n) \cdot \delta
$$

of the identity.

LEMMA 7. Let $f: I^{n} \rightarrow I_{2}^{k} \times I^{q-k}$ be an embedding with

$f\left(I^{n}\right) \cap$ bdy $\left(I_{2}^{k} \times I^{q-k}\right)=I^{k} \times$ bdy $\left(I^{n-k}\right)$,

$f \mid I^{k} \times$ bdy $\left(I^{n-k}\right)$ the identity, and

$f$ within $\delta / 2$ of the identity everywhere.

If $k>0$, assume further that $f \mid C^{1} \cap I^{n}$ is the identity. Then there is an ambient isotopy $\theta_{t}$ of $I_{2}^{k} \times I^{q-k}$ that fixes a neighborhood of the boundary, that moves points less than $\sigma(n, k) \cdot \delta$ (where $\sigma(n, k)$ is a positive real number which depends only on $n$ and $k)$, and such that the pair $\left\{B, \theta_{1} \circ f\left(I^{n}\right) \cap B\right\}$ is homeomorphic to a standard ball pair.

Proof. If $k=0$, Zeeman's unknotting theorem together with the beginning of Alexander's trick gives an ambient isotopy $\theta_{t}^{I}$ of $I^{q}$ that fixes bdy $\left(I^{q}\right)$, that moves 
1.

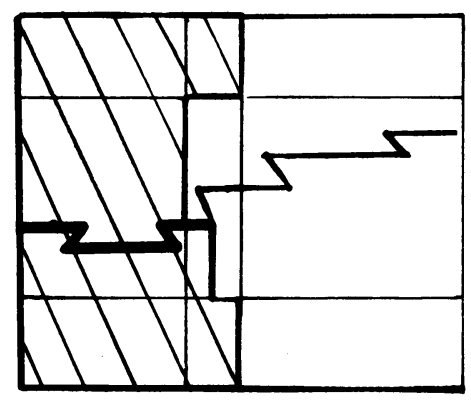

3.

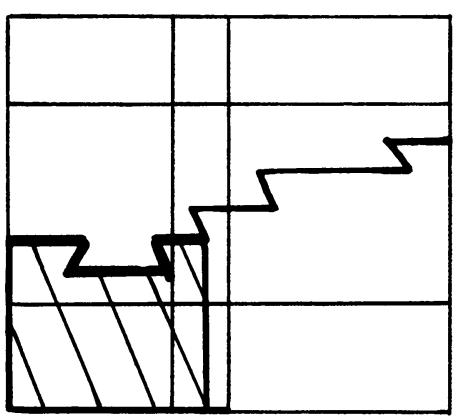

Collapses 1-4 take $S^{14}$ onto $K$ (the heavy line)

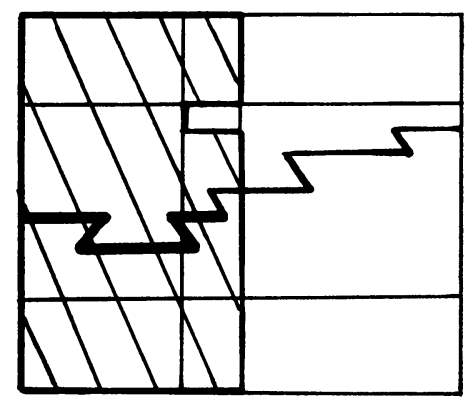

Collapse associated with $\theta^{\text {av }}$
2.

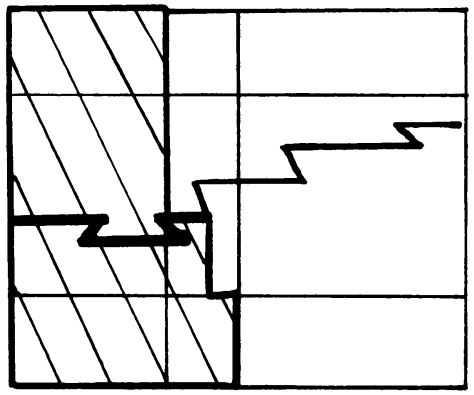

4.

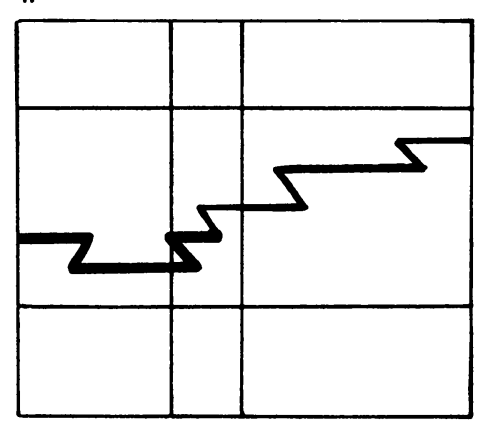

FIGURE 6. Collapses in Lemma 7.

points less than $\delta / 4$, and such that $\theta_{1}^{\mathrm{I}} f$ is the identity on some neighborhood of bdy $\left(I^{n}\right)$ in $I^{n}$. If $\mu$ (the same as in Lemma 5) is chosen small enough, the map $\theta_{1}^{\mathrm{I}} \circ f \mid C^{1} \cap I^{n}$ is the identity. If $k>0$, let $\theta_{t}^{\mathrm{I}}$ be the identity. Thus $\theta_{1}^{\mathrm{I}} \circ f \mid C^{1} \cap I^{n}$ is the identity for all $k$. 
General position gives an ambient isotopy $\theta_{t}^{\mathrm{II}}$ of $I_{2}^{k} \times I^{q-k}$ that fixes

$$
\text { bdy }\left(I_{2}^{k} \times I^{q-k}\right) \cup \theta_{1}^{\mathrm{I}} \circ f\left(C^{1} \cap I^{n}\right),
$$

that moves points less than $\delta / 4$, and such that $\theta_{1}^{\mathrm{II}} \circ \theta_{1}^{\mathrm{I}} \circ f\left(I^{n}\right)$ meets each vertical line finitely and has singular set with respect to vertical projection of dimension $\leqq(n-2)$.

Set $\hat{f} \equiv \theta_{1}^{\text {II }} \circ \theta_{1}^{\mathrm{I}} \circ f$. Then $\hat{f}: I^{n} \rightarrow I_{2}^{k} \times I^{q-k}$ satisfies the hypothesis of Lemma 5 . Let $K$ be the set given in the conclusion of that lemma.

At this point, take subdivisions of $I_{2}^{k} \times I^{q-k}$ and $I^{n}$ such that $\hat{f}$ is simplicial, that the collapse from $K$ to $\hat{f}(v)$ is simplicial, and that the triangulation of $I_{2}^{k} \times I^{q-k}$ is cylindrical in the sunny direction. Take first and second derived subdivisions of these.

If $X$ is a subset of $I_{2}^{k} \times I^{q-k}$, let $X_{s}$ denote the shadow set of $X$; that is, $X$ together with all points shadowed by $X$.

Let $S^{12} \equiv B-\operatorname{int}\left(I_{2}^{k} \times I^{q-k-1} \times I_{\delta}\right)$. Since $\hat{f}$ is within $\delta$ of the identity, $\hat{f}\left(I^{n}\right)$ can intersect $S^{12}$ only in bdy $\left(I_{2}^{k} \times I^{q-k-1}\right) \times I_{\delta}$. The set $S^{12} \cup K_{s} \cup A \cup \hat{f}\left(I^{n}\right)$ collapses to

$$
\left(B \cap\left(I_{2}^{k} \times I^{q-k-1} \times[-1,-\delta] \cup\left(\operatorname{bdy}\left(I_{2}^{k} \times I^{q-k-1}\right) \times I\right)\right) \cup K_{s} \cup A \cup \hat{f}\left(I^{n}\right) .\right.
$$

This collapses to

$$
\left(B \cap\left(\left(I_{2}^{k} \times I^{q-k-1}\right) \times\{-1\}\right)\right) \cup K_{s} \cup \hat{f}\left(I^{n}\right) \equiv S^{13}
$$

by vertical collapses, since

$$
K \supset \hat{f}\left(I^{n}\right) \cap\left(A \cup\left(B \cap\left(\operatorname{bdy}\left(I_{2}^{k} \times I^{q-k-1}\right) \times I\right)\right)\right) .
$$

Zeeman's proof of Theorem 20 shows that $S^{13}$ collapses simplicially to

$$
\left(B \cap\left(\left(I_{2}^{k} \times I^{q-k-1}\right) \times\{-1\}\right)\right) \cup \hat{f}(v)_{s} \cup \hat{f}\left(I^{n}\right) .
$$

This collapses to $\hat{f}\left(I^{n}\right)$. In particular, the set $S^{12} \cup K_{s} \cup A \equiv S^{14}$ is contained in $B$, and it collapses simplicially to $K$ without disturbing $\hat{f}\left(I^{n}\right)$. By Proposition 1, there is a homeomorphism of $N\left(S^{14}, B^{\prime \prime}\right)$ onto $N\left(K, B^{\prime \prime}\right)$ that fixes $\hat{f}\left(I^{n}\right)$. This means that the pair

$$
S^{15} \equiv\left\{N\left(S^{14}, B^{\prime \prime}\right), N\left(S^{14}, B^{\prime \prime}\right) \cap \hat{f}\left(I^{n}\right)\right\}
$$

is homeomorphic to the pair $\left\{N\left(K, B^{\prime \prime}\right), N\left(K, B^{\prime \prime}\right) \cap \hat{f}\left(I^{n}\right)\right\}$. This, however, is equal to the pair

$$
\left\{N\left(K,\left(B \cup \overline{\left(I_{2}^{k} \times I^{q-k}\right)-C^{3}}\right)^{\prime \prime}\right), N\left(K,\left(B \cup\left(\overline{\left(I_{2}^{k} \times I^{q-k}\right)-C^{3}}\right)^{\prime \prime}\right) \cap \hat{f}\left(I^{n}\right)\right\},\right.
$$

since $K \cap$ bdy $(B)$ does not meet $\overline{\left(I_{2}^{k} \times I^{q-k}\right)-C^{3}}$ (see Lemma 5 for the definition of $C^{3}$ ). The last pair is equal to the pair

$$
\left\{N\left(K,\left(B \cup \overline{\left.\left(I_{2}^{k} \times I^{q-k}\right)-C^{3}\right)^{\prime \prime}}\right), N\left(K, \hat{f}\left(\left(B \cap I^{n}\right) \cup \overline{I^{n}-C^{3}}\right)^{\prime \prime}\right)\right\} \equiv S^{16},\right.
$$

since $\hat{f}\left(\left(B \cap I^{n}\right) \cup \overline{I^{n}-C^{3}}\right)=\hat{f}\left(I^{n}\right) \cap\left(B \cup \overline{\left(I_{2}^{k} \times I^{q-k}\right)-C^{3}}\right)$. Each of the elements of the pair $S^{16}$ is a ball because $K$ is collapsible. The second element is properly 
embedded in the first (as manifolds with boundary), since each vertex of $K$ that is in the boundary of $\hat{f}\left(\left(B \cap I^{n}\right) \cup \overline{I^{n}-C^{3}}\right)$ is also in the boundary of

$$
B \cup \overline{\left(I_{2}^{k} \times I^{q-k}\right)-C^{3}} .
$$

Thus, $S^{16}$ is a ball pair. Recall that $S^{15}$ is homeomorphic to the pair $S^{16}$; therefore, it too, is a ball pair.

$K$ does not interesect $I_{2}^{k} \times I^{q-k-1} \times([-1,-\delta] \cup[\delta, 1])$, and consequently there is a number $\gamma \in(0, \delta)$ such that $S^{14}$ is contained in

$$
\left(B \cap\left(I_{2}^{k} \times I^{q-k-1} \times([-1, \gamma] \cup[\delta, 1]) \cup \text { bdy }\left(I_{2}^{k} \times I^{q-k-1}\right) \times I\right)\right) \cup A \equiv S^{17} .
$$

There is a vertical collapse from $S^{17}$ to $S^{14}$. The vertical distance from $\gamma$ to $(-\delta)$ is less than $2 \delta$, and the mesh of the triangulation on $I_{2}^{k} \times I^{q-k}$ is less than $\delta$; hence the collapse has diameter less than $3 \delta$. By Corollary 2, there is an ambient isotopy $\theta_{t}^{\text {III }}$ of $I_{2}^{k} \times I^{q-k}$ that takes $N\left(S^{17}, B^{\prime \prime}\right)$ onto $N\left(S^{14}, B^{\prime \prime}\right)$ and that moves points less than $5 \delta$.

For $k=0$, there is a cyclindrical collapse, parallel to the direction of the first coordinate, that takes $B$ onto $S^{17}$ and that has diameter less than $((n+2) 4 \delta+2 \delta)$. For $k>0$, there is a $c$-radial collapse toward $\{o\} \times I^{n-k}$ (see definition in Lemma 5) that takes $B$ onto $S^{17}$ and that has diameter less than $(k(n+2) 4 \delta+2 \delta)$. Accordingly, by Corollary 2, there is an ambient isotopy $\theta_{t}^{\mathrm{IV}}$ of $I_{2}^{k} \times I^{q-k}$ that takes $B\left(=N\left(B, B^{\prime \prime}\right)\right)$ onto $N\left(S^{17}, B^{\prime \prime}\right)$ and that is within $((k+1)(n+2) 4 \delta+4 \delta)$ of the identity (for all $k$ ).

Both collapses fix bdy $\left(I_{2}^{k} \times I^{q-k}\right)$, and therefore, by Proposition 1,

$$
\left(\theta_{t}^{\mathrm{IV}}\right)^{-1} \circ\left(\theta_{t}^{\mathrm{III}}\right)^{-1} \mid N\left(\operatorname{bdy}\left(I_{2}^{k} \times I^{q-k}\right),\left(I_{2}^{k} \times I^{q-k}\right)^{m}\right)
$$

is the identity. Set $\theta_{t} \equiv\left(\theta_{t}^{\mathrm{IV}}\right)^{-1} \circ\left(\theta_{t}^{\mathrm{III}}\right)^{-1} \circ \theta_{t}^{\mathrm{II}} \circ \theta_{t}^{\mathrm{I}}$. Then $\theta_{t}$ fixes bdy $\left(I_{2}^{k} \times I^{q-k}\right)$; if $k>0$, it actually fixes

$$
N\left(\operatorname{bdy}\left(I_{2}^{k} \times I^{q-k}\right),\left(I_{2}^{k} \times I^{q-k}\right)^{m}\right) .
$$

The ambient isotopy $\theta_{t}$ moves points less than

$$
((k+1)(n+2) 4 \delta+4 \delta)+5 \delta+\delta / 4+\delta / 4 \equiv \sigma(n, k) \cdot \delta .
$$

The pair

$$
\left\{B, \theta_{1} f\left(I^{n}\right) \cap B\right\}
$$

equals the pair $\left\{B,\left(\theta_{1}^{\text {IV }}\right)^{-1} \circ\left(\theta_{1}^{\text {III }}\right)^{-1} \circ \hat{f}\left(I^{n}\right) \cap B\right\}$ which is the image under $\left(\theta_{1}^{\mathrm{IV}}\right)^{-1} \circ\left(\theta_{1}^{\mathrm{III}}\right)^{-1}$ of the pair $S^{15}$. Consequently the last displayed pair is homeomorphic to a ball pair. This completes the proof of Lemma 7.

REMARK 8 (Notation as in Lemma 7). Suppose $k=0$. Then there is a homeomorphism

$$
\Delta: B \cap I^{n} \stackrel{\text { onto }}{\longrightarrow} \theta_{1} \circ f\left(I^{n}\right) \cap B
$$

that is the identity on $B \cap \operatorname{bdy}\left(I^{n}\right)$, and that is within $\tau(n) \cdot \delta$ of the identity everywhere. $\tau(n)$ is a real number greater than $\sigma(n, 0)$. 
Proof. The second derived subdivision of $I^{n}$ may be chosen so that

$$
N\left(B \cap I^{n},\left(\left(B \cap I^{n}\right) \cup \overline{I^{n}-C^{3}}\right)^{\prime \prime}\right)
$$

is the set

$$
\left(B \cap I^{n}\right) \cup\left([0, \nu] \times I_{1-\kappa / 2}^{q-1}\right)=\left([-1,0] \times I^{q-1}\right) \cup\left([0, \nu] \times I_{1-\kappa / 2}^{q-1}\right),
$$

(for some $\nu \in(0, \delta)$ ). Then there is a homeomorphism

$$
\Delta^{\mathrm{I}}: B \cap I^{n} \stackrel{\text { onto }}{\longrightarrow} N\left(B \cap I^{n},\left(\left(B \cap I^{n}\right) \cup \overline{I^{n}-C^{3}}\right)^{\prime \prime}\right)
$$

that fixes $B \cap$ bdy $\left(I^{n}\right)$ and moves points less than $\delta$. Let $S^{18}$ denote the range of $\Delta^{\mathrm{I}}$.

For the map $\hat{f}$ of Lemma 7, Remark 6 gives a homeomorphism

$$
\psi: S^{18} \stackrel{\text { onto }}{\longrightarrow} N\left(\hat{f}^{-1}(K),\left(\left(B \cap I^{n}\right) \cup \overline{I^{n}-C^{3}}\right)^{\prime \prime}\right)
$$

that fixes $S^{18} \cap$ bdy $\left(I^{n}\right)$, and that is within $\lambda(n) \cdot \delta$ of the identity everywhere. Thus we have the homeomorphism

$$
\hat{f} \circ \psi \circ \Delta^{\mathrm{I}}: B \cap I^{n} \stackrel{\text { onto }}{\longrightarrow} N\left(K, \hat{f}\left(\left(B \cap I^{n}\right) \cup \overline{I^{n}-C^{3}}\right)^{\prime \prime}\right) .
$$

The range of $\hat{f} \circ \psi \circ \Delta^{\mathrm{I}}$ is equal to

$$
N\left(K,\left(B \cup \overline{\left.\left(I_{2}^{k} \times I^{q-k}\right)-C^{3}\right)^{\prime \prime}}\right) \cap \hat{f}\left(I^{n}\right)=N\left(S^{14}, B^{\prime \prime}\right) \cap \hat{f}\left(I^{n}\right) .\right.
$$

(See the proof of Lemma 7 for a discussion of these equalities.) The last paragraph of the proof of Lemma 7 shows that

$$
\left(\theta_{1}^{\mathrm{IV}}\right)^{-1} \circ\left(\theta_{1}^{\mathrm{III}}\right)^{-1}\left(N\left(S^{14}, B^{\prime \prime}\right) \cap \hat{f}\left(I^{n}\right)\right)=\theta_{1} \circ f\left(I^{n}\right) \cap B .
$$

Set $\Delta=\theta_{1} \circ \hat{f} \circ \psi \circ \Delta^{\mathrm{I}}$. Then $\Delta: B \cap I^{n} \rightarrow \theta_{1} f\left(I^{n}\right) \cap B$, fixes $B \cap$ bdy $\left(I^{n}\right)$, and is within $(\sigma(n) \cdot \delta+\delta+\lambda(n) \cdot \delta+\delta)=\tau(n) \cdot \delta$ of the identity.

THEOREM $9(q-n, n)$. For $q-n \geqq 3$ and $\varepsilon>0$, there is a $\delta>0$ such that iff: $I^{n} \rightarrow I^{q}$ is a proper embedding with $f \mid \mathrm{bdy}\left(I^{n}\right)$ the identity and with $f$ within $\delta$ of the identity everywhere, then there is an ambient isotopy of $I^{q}$ that fixes bdy $\left(I^{q}\right)$, that moves points less than $\varepsilon$, and that takes $f$ to the identity.

Suppose $w$ is a positive integer. Partition $I^{n}$ into $w^{n}$ congruent cells $C_{1}, \ldots, C_{w^{n}}$, each being similar to $I^{n}$. Arrange the ordering so that $\bigcup_{i=1}^{r-1} C_{i}$ is a cell that intersects $C_{r}$ in an $(n-1)$-cell (for each $\left.r=1, \ldots, w^{n}\right)$.

LeMma $10(q-n, n, w, r)$. There is $a \delta_{r}>0$ such that if

$$
f: \bigcup_{i=1}^{r} C_{i} \rightarrow\left(\bigcup_{i=1}^{r} C_{i}\right) \times I^{q-n}
$$

is a proper embedding with

$$
f \mid \operatorname{bdy}\left(\bigcup_{i=1}^{r} C_{i}\right)=\text { the identity, }
$$


and with $f$ within $\delta_{r}$ of the identity everywhere, then there is an ambient isotopy of $\bigcup_{i=1}^{r} C_{i} \times I^{q-n}$ that fixes bdy $\left(\bigcup_{i=1}^{r} C_{i} \times I^{q-n}\right)$, that moves points less than $2 q / w$, and that takes the set $f\left(\bigcup_{i=1}^{r} C_{i}\right)$ onto the set $\bigcup_{i=1}^{r} C_{i}$.

For a fixed codimension $q-n \geqq 3$, we prove Theorem 9 and Lemma 10 by the following induction.

1. Lemma $10(q-n, n, w, 1)$.

2. Theorem $9(q-n, n-1)$ together with Lemma $10(q-n, n, w, r-1)$ imply Lemma $10(q-n, n, w, r)$. Induction on $r$ then implies Lemma $10\left(q-n, n, w, w^{n}\right)$.

3. If $\varepsilon>2 q / w$, then Lemma $10\left(q-n, n, w, w^{n}\right)$ implies Theorem $9(q-n, n)$. For any $\varepsilon>0$, there is some $w$ with $\varepsilon>2 q / w$, and this implies Theorem $9(q-n, n)$.

The induction starts trivially at Theorem $9(q-n, 0)$.

Proof. 1. Choose $\delta_{1}<1 / w$. Then $f$ actually embeds $C_{1}$ in $C_{1} \times I_{1 / w}^{q-n}$. This embedding is proper, and $f \mid$ bdy $\left(C_{1}\right)$ is the identity. Thus, by Zeeman's unknotting theorem, there is an ambient isotopy of $C_{1} \times I_{1 / w}^{q-n}$ which fixes bdy $\left(C_{1} \times I_{1 / w}^{q-n}\right)$ and takes $f$ to the identity. Since the diameter of $C_{1} \times I_{1 / w}^{q-n}$ is less than $2 q / w$, the ambient isotopy is automatically within $2 q / w$ of the identity. Since it fixes the boundary of $C_{1} \times I_{1 / w}^{q-n}$, it extends, by the identity, to an ambient isotopy of $C_{1}$ $\times I^{q-n}$ that fixes bdy $\left(C_{1} \times I^{q-n}\right)$, that moves points less than $2 q / w$, and that takes $f$ to the identity.

2. Suppose Theorem $9(q-n, n-1)$ and Lemma $10(q-n, n, w, r-1)$ are already proved. Let $\eta^{\mathrm{I}}: \bigcup_{i=1}^{r} C_{i} \stackrel{\text { onto }}{\longrightarrow} I^{n}$ be a homeomorphism that takes $\bigcup_{i=1}^{r-1} C_{i}$ onto $[-1,0] \times I^{n-1}$ and takes $C_{r}$ onto $[0,1] \times I^{n-1}$. Define

$$
\eta^{\mathrm{II}}=\eta^{\mathrm{I}} \times \mathrm{id}: \bigcup_{i=1}^{r} C_{i} \times I^{q-n} \stackrel{\text { onto }}{\longrightarrow} I^{q} .
$$

Since the domain of $\eta^{\mathrm{II}}$ is compact, there is a number $\alpha>1$ such that

$$
\frac{1}{\alpha}<\frac{\text { distance }\left(\eta^{\mathrm{II}}(x), \eta^{\mathrm{II}}(y)\right)}{\operatorname{distance}(x, y)}<\alpha
$$

(for all $x \neq y$ in $\bigcup_{i=1}^{r} C_{i} \times I^{q-n}$ ).

Let $\delta_{n-1}$ be $\delta_{r-1} / 3 \alpha$ or the $\delta$ in Theorem $9(q-n, n-1)$ for which the ambient isotopy there is within $\delta_{r-1} / 3 \alpha$ of the identity, whichever is smaller. Choose $\delta_{r}$ such that $0<\delta_{r}<\delta_{r-1} / 3$ and such that $\delta_{r} \cdot \alpha \cdot \tau(n)<\delta_{n-1}$.

Let $f: \bigcup_{i=1}^{r} C_{i} \rightarrow \bigcup_{i=1}^{r} C_{i} \times I^{q-n}$ be a proper embedding such that

$$
f \mid \operatorname{bdy}\left(\bigcup_{i=1}^{r} C_{i}\right)
$$

is the identity and such that $f$ is within $\delta_{r}$ of the identity everywhere. Then $\eta^{\mathrm{II}} \circ f \circ \eta^{\mathrm{I}}: I^{n} \rightarrow I^{q}$ is a proper embedding whose restriction to the boundary is the identity and which is within $\alpha \cdot \delta_{r}$ of the identity everywhere. By Lemma 7.(k=0), there is an ambient isotopy $\theta_{t}$ of $I^{q}$ that fixes bdy $\left(I^{q}\right)$, that moves points less than 
$\alpha \cdot \delta_{r} \cdot \sigma(n, 0)$, and such that the pair $\left\{B, \theta_{1} \circ f\left(I^{n}\right) \cap B\right\}$ is a ball pair. By Remark 8, there is a homeomorphism

$$
\Delta: B \cap I^{n} \stackrel{\text { onto }}{\longrightarrow} \theta_{1} \circ f\left(I^{n}\right) \cap B
$$

that is the identity on $B \cap$ bdy $\left(I^{n}\right)$, and such that $\Delta$ is within $\alpha \cdot \delta_{r} \cdot \tau(n)$ of the identity everywhere. In particular,

$$
\Delta \mid B \cap \overline{\left(I^{n}-B\right)}: B \cap \overline{\left(I^{n}-B\right)} \stackrel{\text { onto }}{\longrightarrow} \theta_{1} \circ f\left(I^{n}\right) \cap B \cap \overline{\left(I^{q}-B\right)}
$$

is the identity on bdy $\left(B \cap \overline{I^{n}-B}\right)$ and is within $\alpha \cdot \delta_{r} \cdot \tau(n)\left(<\delta_{n-1}\right)$ of the identity everywhere. Since $B \cap \overline{I^{n}-B}=\{0\} \times I^{n-1}$, and since $B \cap \overline{I^{q}-B}=\{0\} \times I^{q-1}$, Theorem $9(q-n, n-1)$ applies to $\Delta \mid B \cap \overline{I^{n}-B}$, and yields an ambient isotopy $\theta_{t}^{n-1}$ of $\{0\} \times I^{q-1}$ that fixes bdy $\left(\{0\} \times I^{q-1}\right)$, that moves points less than $\delta_{r-1} / 3 \alpha$, and such that $\theta_{1}^{n-1} \circ \Delta \mid\{0\} \times I^{n-1}$ is the identity. $\theta_{t}^{n-1}$ extends by collaring to $\theta_{t}^{\mathrm{v}}$, an ambient isotopy of $I^{q}$ that fixes bdy $\left(I^{q}\right)$, that preserves $B$ and $\overline{I^{q}-B}$, and that moves points less than $\delta_{r-1} / 3 \alpha$.

$\theta_{1}^{\mathrm{v}} \circ \Delta: B \cap I^{n} \rightarrow B$ is a proper embedding with $\theta_{1}^{\mathrm{v}} \circ \Delta /$ bdy $\left(B \cap I^{n}\right)$ the identity, and with $\theta_{1}^{\mathrm{V}} \circ \Delta$ within $\left(\alpha \cdot \delta_{r} \cdot \tau(n)+\delta_{r-1} / 3 \alpha\right)\left(<\frac{2}{3} \cdot \delta_{r-1} / \alpha\right)$ of the identity everywhere. Thus

$$
\left(\eta^{\mathrm{II}}\right)^{-1} \circ \theta_{1}^{\mathrm{V}} \circ \Delta \circ \eta^{\mathrm{I}}: \bigcup_{i=1}^{r-1} C_{i} \stackrel{\text { onto }}{\longrightarrow}\left(\left(\eta^{\mathrm{II}}\right)^{-1} \circ \theta_{1}^{\mathrm{V}} \circ \theta_{1} \circ \eta^{\mathrm{I}} f\left(\bigcup_{i=1}^{r} C_{i}\right)\right) \cap \bigcup_{i=1}^{r-1} C_{i} \times I^{q-n}
$$

is a proper embedding of $\bigcup_{i=1}^{r-1} C_{i}$ into $\bigcup_{i=1}^{r-1} C_{i} \times I^{q-n}$ that is the identity on bdy $\left(\cup_{i=1}^{r-1} C_{i}\right)$ and that is within $\frac{2}{3} \cdot \delta_{r-1}$ of the identity everywhere. By Lemma 10 $(q-n, n, w, r-1)$, there is an ambient isotopy $\theta_{t}^{\mathrm{VI}}$ of $\bigcup_{i=1}^{r-1} C_{i} \times I^{q-n}$ that fixes bdy $\left(\bigcup_{i=1}^{r-1} C_{i} \times I^{q-n}\right)$, that moves points less than $2 q / w$, and such that

$$
\theta_{1}^{\mathrm{VI}} \circ\left(\eta^{\mathrm{II}}\right)^{-1} \circ \theta_{1}^{\mathrm{V}} \circ \Delta \circ \eta^{\mathrm{I}}\left(\bigcup_{i=1}^{r-1} C_{i}\right)=\bigcup_{i=1}^{r-1} C_{i}
$$

The ambient isotopy $\theta_{t}^{\mathrm{vI}}$ extends by the identity to an ambient isotopy, also called $\theta_{t}^{\mathrm{vI}}$, on $\bigcup_{i=1}^{r} C_{i} \times I^{q-n}$. Consequently

$$
\begin{aligned}
S^{19} & \equiv\left(\theta_{1}^{\mathrm{VI}} \circ\left(\eta^{\mathrm{II}}\right)^{-1} \circ \theta_{1}^{\mathrm{V}} \circ \theta_{1} \circ \eta^{\mathrm{I}} \circ f\left(\bigcup_{i=1}^{r} C_{i}\right)\right) \cap\left(C_{r} \times I^{q-n}\right) \\
& =\left(\left(\eta^{\mathrm{II}}\right)^{-1} \circ \theta_{1}^{\mathrm{V}} \circ \theta_{1} \circ \eta^{\mathrm{I}} \circ f\left(\bigcup_{i=1}^{r} C_{i}\right)\right) \cap\left(C_{r} \times I^{q-n}\right) .
\end{aligned}
$$

The last set is an $n$-cell properly embedded in $C_{r} \times I^{q-n}$. The boundary of the $n$-cell is the set bdy $\left(C_{r}\right) \times\{0\}$. Since $f$ is within $\delta_{r-1} / 3$ of the identity, and since the map $\left(\eta^{\mathrm{II}}\right)^{-1} \circ\left(\theta_{1}^{\mathrm{V}} \circ \theta_{1}\right) \cdot \eta^{\mathrm{I}}$ is within $\alpha \cdot \frac{2}{3} \cdot \delta_{r-1} / \alpha$ of the identity, the map

$$
\left(\eta^{\mathrm{II}}\right)^{-1} \circ \theta_{1}^{\mathrm{V}} \circ \theta_{1} \circ \eta^{\mathrm{I}} \circ f
$$

is within $\delta_{r-1}$ of the identity. Recall that $\delta_{r-1}<1 / w$. Thus $S^{19}$ is properly embedded in $C_{r} \times I^{q-n}$. Part 1 of this proof now gives an ambient isotopy $\theta_{t}^{\mathrm{VII}}$ of $C_{r} \times I^{q-n}$ that fixes bdy $\left(C_{r} \times I^{q-n}\right)$, that moves points less than $2 q / w$, and that takes $S^{19}$ 
onto $C_{r} \times\{0\}$. $\theta_{t}^{\mathrm{vII}}$ extends by the identity to an ambient isotopy, also called $\theta_{t}^{\mathrm{vII}}$, of $\bigcup_{i=1}^{r} C_{i} \times I^{q-n}$

$\theta_{t}^{\mathrm{VII}} \circ \theta_{t}^{\mathrm{VI}} \circ\left(\eta^{\mathrm{II}}\right)^{-1} \circ \theta_{t}^{\mathrm{V}} \circ \theta_{t} \circ \eta^{\mathrm{I}}$ is the ambient isotopy that completes the proof of Lemma $10(q-n, n-w, r)$.

3. This part depends on Alexander's trick for showing that a homeomorphism of a cell that fixes the boundary is ambient isotopic to the identity. Let $\tilde{f}: I^{n} \stackrel{\text { onto }}{\longrightarrow} I^{n}$ be a homeomorphism that is the identity on bdy $\left(I^{n}\right)$. Let $\nu=(0, \ldots, 0,1) \in I^{n}$ $\times[0,1]$. Extend $\tilde{f}$ first to $C \tilde{f}: \nu \cdot I^{n} \stackrel{\text { onto }}{\longrightarrow} \nu \cdot I^{n}$ by mapping the base of the cone by $\tilde{f}$, mapping the vertex $\nu$ to itself, and extending linearly. $C f^{f} \mid \nu \cdot$ bdy $\left(I^{n}\right)$ is the identity, and consequently, $C \tilde{f}$ extends, by the identity, to $\tilde{F}: I^{n} \times[0,1] \stackrel{\text { onto }}{\longrightarrow} I^{n} \times[0,1] . \tilde{F}$ is an ambient isotopy of $I^{n}$ which has the property that if $\tilde{f}^{\tilde{f}}$ is within $\tilde{\varepsilon}$ of the identity everywhere, then so is $\widetilde{F}$. Set $\widetilde{F}_{t}(x)=\widetilde{F}(x, t)$.

Lemma $10\left(q-n, n, w, w^{n}\right)$ yields a number $\delta_{w^{n}}>0$ such that if $f: I^{n} \rightarrow I^{n} \times I^{q-n}$ is a proper embedding with $f \mid$ bdy $\left(I^{n}\right)$ the identity and with $f$ within minimum $\left\{\delta_{w^{n}}, 2 q / w\right\}$ of the identity everywhere, then there is an ambient isotopy $\theta_{t}^{\mathrm{VIII}}$ of $I^{q}$

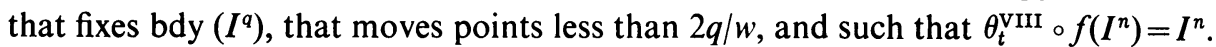

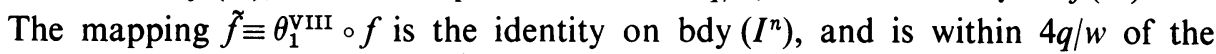
identity everywhere. For $\tilde{F}_{t}$ defined above, $\widetilde{F}_{1} \circ \theta_{1}^{\text {vIII }} \circ f$ is the identity. The isotopy $\tilde{F}_{t}$ extends by $(q-n)$-fold collaring to an ambient isotopy $\tilde{F}_{t}$ of $I^{q}$ that fixes bdy $\left(I^{q}\right)$, and that moves points less than $4 q / w$.

If we choose $w$ so that $\varepsilon>6 q / w$, then $\tilde{F}_{t} \circ \theta_{t}^{\mathrm{vIII}}$ is the ambient isotopy required in Theorem $9(q-n, n)$.

THEOREM $11(q-n, n)$. Let $M$ be a compact $n$-manifold, $\hat{M}$ a compact $n$-submanifold of $M, Q$ a $q$-manifold, and $n \leqq q-3$. Let $g: M \rightarrow Q$ be an embedding such that $g(M) \subset \operatorname{int}(Q)$. Let $N$ be a neighborhood of $g(\overline{M-\hat{M})}$ in $Q$. Then for $\varepsilon>0$, there is $a \delta>0$ such that if $h: M \rightarrow Q$ is an embedding with $h(M) \subset \operatorname{int}(Q)$, with $h|\hat{M}=g| \hat{M}$, and with $h$ within $\delta$ of $g$ everywhere, then there is an ambient isotopy of $Q$ that is fixed outside $N$, that moves points less than $\varepsilon$, and that takes $h$ to $g$.

LEMmA $12(q-n, n)$. Suppose $0 \leqq k \leqq n \leqq q-3$ and $\varepsilon>0$. Then there is a $\delta>0$ such that if $f: I^{n} \rightarrow I_{2}^{k} \times I^{q-k}$ is an embedding with

$f\left(I^{n}\right) \cap$ bdy $\left(I_{2}^{k} \times I^{q-k}\right)=I^{k} \times$ bdy $\left(I^{n-k}\right)$,

$f \mid \overline{I^{n}-\left(I^{k} \times I_{1 / 2}^{n-k}\right)}$ the identity, and

$f$ within $\delta$ of the identity everywhere, then there is an ambient isotopy of $I_{2}^{k} \times I^{q-k}$ that fixes bdy $\left(I_{2}^{k} \times I^{q-k}\right)$, that moves points less than $\varepsilon$, and that takes $f$ to the identity.

Fix a codimension $(q-n)$. If $k=0$, Theorem $9(q-n, n)$ implies Lemma 12 $(q-n, n)$ immediately. If $k>0$, we prove Lemma 12 and Theorem 11 by the following induction:

1. Theorem $11(q-n, n-1)$ implies Lemma $12(q-n, n)$.

2. Lemma $12(q-n, n)$ implies Theorem $11(q-n, n)$.

The induction starts trivially with Lemma $12(q-n, 0)$. 
Proof. 1. $\varepsilon>0$ is given. Choose $\varepsilon^{\text {thm } 9}$ such that $2\left(\varepsilon^{\text {thm } 9}\right)<\varepsilon$. Theorem 9 associates a $\delta^{\text {thm } 9}$ to $\varepsilon^{\text {thm } 9}$. Choose $\varepsilon^{\text {thm } 11}$ such that $2\left(\varepsilon^{\text {thm } 11}\right)<\operatorname{minimum}\left\{\delta^{\text {thm } 9}, \varepsilon^{\text {thm } 9}\right\}$. Theorem $11(q-n, n-1)$ associates a $\delta^{\text {thm } 11}$ to $\varepsilon^{\text {thm } 11}$. Choose $\delta$ such that

$$
(\sigma(n, k) \cdot \delta+k(n+2) 4 \delta+4 \delta+2 \delta)<\min \left\{\delta^{\mathrm{thm} 11}, \varepsilon^{\mathrm{thm} 11}\right\} .
$$

Finally choose $\delta=\delta / 2$.

The hypotheses for Lemma $12(q-n, n)$ are precisely those of Lemma 7 where $\delta=\delta / 2$. By Lemma 7 , there is a set $K$ and an ambient isotopy $\theta_{t}$ of $I_{2}^{k} \times I^{q-k}$ such that $\left\{B, \theta_{1} \circ f\left(I^{n}\right) \cap B\right\}$ is a ball pair and

$$
\begin{aligned}
\theta_{1} \circ f\left(I^{n}\right) \cap B & =\left(\theta_{1}^{\mathrm{IV}}\right)^{-1} \circ\left(\theta_{1}^{\mathrm{III}}\right)^{-1}\left(N\left(S^{14}, B^{\prime \prime}\right) \cap \hat{f}\left(I^{n}\right)\right) \\
& =\left(\theta_{1}^{\mathrm{IV}}\right)^{-1} \circ\left(\theta_{1}^{\mathrm{III}}\right)^{-1}\left(N\left(K, \hat{f}\left(\left(B \cap I^{n}\right) \cup \overline{I^{n}-C^{3}}\right)^{\prime \prime}\right)\right) \\
& =\left(\theta_{1}^{\mathrm{IV}}\right)^{-1} \circ\left(\theta_{1}^{\mathrm{III}}\right)^{-1}\left(N\left(K, \hat{f}\left(I^{n}\right)^{\prime \prime}\right)\right) .
\end{aligned}
$$

The last equality holds since $\left(B \cap I^{n}\right) \cup \overline{I^{n}-C^{3}}=I^{n}$ if $k>0$. (Compare this with the next to the last paragraph of the proof of Remark 8.)

$\hat{f}\left(I^{n}\right)$ is a submanifold of $I_{2}^{k} \times I^{q-k}$ of the sort required in Proposition $1 . K$ is a subcomplex of $\hat{f}\left(I^{n}\right)$, and $\hat{f}\left(I^{n}\right)$ collapses to $K$ by a collapse whose diameter is less than $(k(n+2) 4 \delta+2 \delta)$. This number comes from Remark 4 , together with the paragraph in Lemma $5(k>0)$ where $c$-radial collapse is defined. By Corollary 2 there is an ambient isotopy $\Xi_{t}^{\mathrm{I}}$ of $I_{2}^{k} \times I^{q-k}$ that fixes a neighborhood of the boundary, that moves points less than $(k(n+2) 4 \delta+4 \delta)$, and such that

$$
\Xi_{1}^{\mathrm{I}} \circ \hat{f}\left(I^{n}\right)=N\left(K, \hat{f}\left(I^{n}\right)^{\prime \prime}\right) .
$$

This, combined with the second paragraph of this part, shows that $\theta_{1} \circ \Xi_{1}^{\mathrm{I}} \circ f\left(I^{n}\right)$ is properly embedded in $I^{q}(=B)$. This embedding induces an embedding of bdy $\left(I^{n}\right)$ into bdy $\left(I^{q}\right)$ that is the identity on a neighborhood of $I^{k} \times$ bdy $\left(I^{n-k}\right)$ in bdy $\left(I^{n}\right)$, and that is within $(\sigma(n, k) \cdot \delta+k(n+2) 4 \delta+4 \delta+2 \delta)$ of the identity everywhere. This number is less than minimum $\left\{\delta^{\text {thm } 11}, \varepsilon^{\text {thm } 11}\right\}$. Thus Theorem 11 applies to

$$
\theta_{1} \circ \Xi_{1}^{\mathrm{I}} \circ f: \operatorname{bdy}\left(I^{n}\right) \rightarrow \operatorname{bdy}\left(I^{q}\right)
$$

to yield an ambient isotopy $\Xi_{t}^{\text {II }}$ of bdy $\left(I^{q}\right)$ that is fixed on a neighborhood of bdy $\left(I^{q}\right) \cap$ bdy $\left(I_{2}^{k} \times I^{q-k}\right)$ in bdy $\left(I^{q}\right)$, that moves points less than $\varepsilon^{\text {thm } 11}$, and such that $\Xi_{1}^{\mathrm{II}} \circ \theta_{1} \circ \Xi_{1}^{\mathrm{I}} \circ f \mid$ bdy $\left(I^{n}\right)$ is the identity. The isotopy $\Xi_{t}^{\mathrm{II}}$ extends by collaring to an ambient isotopy of $I_{2}^{k} \times I^{q-k}$, also called $\Xi_{t}^{\mathrm{II}}$, that fixes a neighborhood of bdy $\left(I_{2}^{k} \times I^{q-k}\right)$, that satisfies $\Xi_{t}^{\mathrm{II}}\left(I^{q}\right)=I^{q}$, and that moves points less than $\varepsilon^{\text {thm } 11}$. It is still true that $\Xi_{1}^{\mathrm{II}} \circ \theta_{1} \circ \Xi_{1}^{\mathrm{I}} \circ f \mid$ bdy $\left(I^{n}\right)$ is the identity. In addition,

$$
\Xi_{1}^{\mathrm{II}} \circ \theta_{1} \circ \Xi_{1}^{\mathrm{I}} \circ f: I^{n} \rightarrow I^{q}
$$

is a proper embedding and is within $\delta^{\text {thm } 9}$ of the identity everywhere. By Theorem 9 , there is an ambient isotopy $\Xi_{t}^{\mathrm{III}}$ of $I^{q}$ that fixes bdy $\left(I^{q}\right)$, that moves points less than $\varepsilon^{\text {thm } 9}$, and such that $\Xi_{1}^{\mathrm{III}} \circ \Xi_{1}^{\mathrm{II}} \circ \theta_{1} \circ \Xi^{\mathrm{I}} \circ f$ is the identity. The ambient isotopy $\Xi_{t}^{\text {III }}$ extends by the identity to the ambient isotopy $\Xi_{t}^{\text {III }}$ on $I_{2}^{k} \times I^{q-k}$. Define 
$\Xi_{t}=\Xi_{t}^{\mathrm{III}} \circ \Xi_{t}^{\mathrm{II}} \circ \theta_{t} \circ \Xi_{t}^{\mathrm{I}}$. Then $\Xi_{1} \circ f$ is the identity, $\Xi_{t}$ fixes bdy $\left(I_{2}^{k} \times I^{q-k}\right)$, and $\Xi_{t}$ moves points less than $\varepsilon$.

A compact $n$-manifold may be built up through a finite sequence of $n$-manifolds, the first being a submanifold, and each subsequent element of the sequence obtained by attaching an $n$-cell to the previous element by identifying an unknotted solid torus $\left(B^{k} \times S^{n-k}\right)$ in the boundary of the cell with a solid torus in the boundary of the previous element. Such a sequence is called a handle decomposition of the manifold.

By induction on the number of elements in the handle decomposition of $M$, it is sufficient to prove Theorem $11(q-n, n)$ for the special case that $M=\overline{M-H} \cup H$ (where $H$ is an $n$-cell, $\overline{M-H}$ is an $n$-manifold, and $\overline{M-H} \cap H$ is a solid torus), that $N$ is a neighborhood of $H$ in $Q$, and that $g|\overline{M-H}=h| \overline{M-H}$.

2. Let $\lambda^{\mathrm{I}}: I^{k} \times I_{3 / 2}^{n-k} \stackrel{\text { onto }}{\longrightarrow} N\left(N\left(H, M^{\prime \prime}\right), M^{\prime \prime}\right)$ be a homeomorphism such that $\lambda^{\mathrm{I}}\left(I^{k} \times I^{n-k}\right)=N\left(H, M^{\prime \prime}\right)$ and $\lambda^{\mathrm{I}}\left(I^{k} \times I_{1 / 2}^{n-k}\right)=H$. Construct, as follows, a homeomorphism $\lambda$ that extends $g \circ \lambda^{\mathrm{I}} \mid I^{k} \times I^{n-k}$, and maps $I_{2}^{k} \times I^{q-k}$ onto a neighborhood of $g(H)$ in $Q$. We lose no generality by assuming that $g\left(N\left(N\left(H, M^{\prime \prime}\right), M^{\prime \prime}\right)\right)$ is contained in $N$. Let $\hat{N}$ be any regular neighborhood of $g\left(N\left(N\left(H, M^{\prime \prime}\right), M^{\prime \prime}\right)\right)$ that is contained in $N$. By homogeneity, there is a homeomorphism $\lambda^{\mathrm{II}}$ that maps the pair

$$
\left\{\hat{N}, g\left(N\left(N\left(H, M^{\prime \prime}\right), M^{\prime \prime \prime}\right)\right)\right\}
$$

onto the pair $\left\{I_{2}^{q}, I^{k} \times I_{3 / 2}^{n-k}\right\}$. Then the composition

$$
\lambda^{\mathrm{II}} \circ g \circ \lambda^{\mathrm{I}}: I^{k} \times I_{3 / 2}^{n-k} \stackrel{\text { onto }}{\longrightarrow} I^{k} \times I_{3 / 2}^{n-k} .
$$

Let $\lambda^{\mathrm{III}}: I_{2}^{n} \stackrel{\text { onto }}{\longrightarrow} I_{2}^{n}$ be any extension of $\lambda^{\mathrm{II}} \circ g \circ \lambda^{\mathrm{I}}$, and let

$$
\lambda^{\mathrm{IV}}=\lambda^{\mathrm{III}} \times \mathrm{id}: I_{2}^{n} \times I_{2}^{q-n}=I_{2}^{q} \stackrel{\text { onto }}{\longrightarrow} I_{2}^{q} .
$$

Then the homeomorphism $\lambda^{\mathrm{v}}=\left(\lambda^{\mathrm{II}}\right)^{-1} \circ \lambda^{\mathrm{Iv}}: I_{2}^{q} \stackrel{\text { onto }}{\longrightarrow} \hat{N}$ extends $g \circ \lambda^{\mathrm{I}}$. Thus, if $\mu$ is chosen small enough,

$$
\hat{N} \equiv \lambda^{\mathrm{V}}\left(I_{1+\mu}^{k} \times I^{n-k} \times I_{\mu}^{q-n}\right) \cap g(M)=g \circ \lambda^{\mathrm{I}}\left(I^{n}\right) .
$$

Let $\lambda^{\mathrm{vI}}: I_{2}^{k} \times I^{n-k} \times I^{q-n} \stackrel{\text { onto }}{\longrightarrow} I_{1+\mu}^{k} \times I^{n-k} \times I_{\mu}^{q-n}$ extend the identity map on $I^{k} \times I^{n-k}$. Then the composition $\lambda=\lambda^{\mathrm{v}} \circ \lambda^{\mathrm{vI}}$ is the required map.

Let $\alpha>1$ be a real number such that

$$
\frac{1}{\alpha}<\frac{\text { distance }(\lambda(x), \lambda(y))}{\text { distance }(x, y)}<\alpha
$$

(for all $x \neq y$ in $I^{q}$ ). There is a number $\kappa>0$ such that if $h: M \rightarrow$ int $(Q)$ is within $\kappa$ of $g$ everywhere, and if $h|\overline{M-H}=g| \overline{M-H}$, then $h\left(N\left(H, M^{\prime \prime}\right)\right)$ is contained in $N$; this means that $f \equiv \lambda^{-1} \circ h \circ \lambda^{\mathrm{I}}$ is defined. The number $\varepsilon>0$ is given. Let $\delta^{\mathrm{Lem} 12}$ be the number associated to $\varepsilon / \alpha$ by Lemma 12. Choose $\delta<\kappa$ and such that $\alpha \delta<\delta^{\text {Lem 12 }}$. Then, the homeomorphism $f$ satisfies the hypothesis of Lemma 12 
$(q-n, n)$ and is within $\alpha \delta$ of the identity. By Lemma 12, there is an ambient isotopy $\Xi_{t}^{\text {IV }}$ of $I^{q}$ that fixes bdy $\left(I^{q}\right)$, that moves points less than $\varepsilon / \alpha$, and such that $\Xi_{1}^{\text {IV }} \circ f$ is the identity on $I_{2}^{k} \times I^{n-k}$. Define $\Xi_{t}^{\mathrm{V}}=\lambda \circ \Xi^{\mathrm{IV}} \circ \lambda^{-1}$. Then $\Xi_{t}^{\mathrm{V}}$ is an ambient isotopy of $\hat{N}$ that fixes bdy $(\hat{N})$ and moves points less than $\varepsilon$. It extends by the identity to all of $Q$. In addition,

$$
\begin{aligned}
\Xi_{1}^{\mathrm{v}} \circ h \mid N\left(H, M^{\prime \prime}\right) & =\Xi_{1}^{\mathrm{v}} \circ h \circ \lambda^{\mathrm{I}} \circ\left(\lambda^{\mathrm{I}}\right)^{-1} \mid N\left(H, M^{\prime \prime}\right) \\
& =\lambda \circ \Xi_{1}^{\mathrm{IV}} \circ \lambda^{-1} \circ h \circ \lambda^{\mathrm{I}} \circ\left(\lambda^{\mathrm{I}}\right)^{-1} \mid N\left(H, M^{\prime \prime}\right) \\
& =\lambda \circ\left(\lambda^{\mathrm{I}}\right)^{-1} \mid N\left(H, M^{\prime \prime}\right) \\
& =g \mid N\left(H, M^{\prime \prime}\right) .
\end{aligned}
$$

This completes the proof of Part 2.

From this point on, maps are necessarily p.l. only if so noted.

PROPOSITION 13. If $G$ is a homeomorphism of $R^{q}$ onto itself and $\varepsilon: R^{q} \rightarrow(0, \infty)$ is a continuous function, then there is an ambient isotopy $\beta_{t}$ of $R^{q}$ such that $\beta_{1} \circ G$ is a piecewise-linear homeomorphism and

$$
\text { distance }\left(\beta_{t}(x), x\right)<\varepsilon(x) \text { for all } x \in R^{q} \text {. }
$$

Proof. Since $q \geqq 5$, either $G$ or $G \circ(\operatorname{Ref})$ is stable where Ref: $R^{q} \rightarrow R^{q}$ is the reflection through the first coordinate. (See [6].)

Connell [3] and Bing [1] have shown that a stable homeomorphism of $R^{q}$ onto itself, $q \geqq 5$, can be ambient isotoped to a piecewise-linear one that is an approximation in the sense of the statement of this proposition. Let $\beta_{t}$ be such an ambient isotopy. If $G$ is stable, we are done. If $G \circ(\operatorname{Ref})$ is stable, then $\beta_{1} \circ G \circ(\operatorname{Ref})$ is piecewise-linear and so,

$$
\beta_{1} \circ G=\beta_{1} \circ G \circ(\operatorname{Ref}) \circ(\operatorname{Ref})^{-1}
$$

is piecewise-linear, which completes the proof of the proposition.

Proof of Theorem 14. Since $M$ is a compact p.l. manifold, and $\hat{M}$ is a compact p.l. $n$-submanifold, there is a finite handle decomposition of $M$ that begins with $\hat{M}$ and builds up to $M$. We may assume that the individual handles are so small that $g$ restricted to a neighborhood of each is actually flat. By induction on the number of handles, Theorem 14 reduces to the special case that $M=\overline{M-H} \cup H$ where $H$ is an $n$-cell, $\overline{M-H}=\hat{M}$ is a compact p.l. $n$-manifold, $\hat{M} \cap H$ is p.l. homeomorphic to $S^{k-1} \times B^{n-k}$ for some $0<k \leqq n, g$ restricted to a neighborhood of $H$ in $M$ is flat, and $N$ is a copy of $R^{q}$ that contains $H$.

Subdivide $M$ and $Q$ so that in the new triangulation $g \mid \hat{M}$ is simplicial, so that

$$
g\left(N\left(N\left(H, M^{\prime \prime}\right), M^{\prime \prime}\right)\right) \subset N,
$$

and so that $g \mid N\left(N\left(H, M^{\prime \prime}\right), M^{\prime \prime}\right)$ is flat. This last condition implies the existence of a homeomorphism $G: N\left(N\left(H, M^{\prime \prime}\right), M^{\prime \prime \prime}\right) \times I^{q-n} \stackrel{\text { onto }}{\longrightarrow} N$ that extends $g$. Let $\hat{N}$ denote the image of $G$. 
Now int $(\hat{N})$ is a copy of $R^{q}$ and has a piecewise-linear structure induced by that on $Q$. Let

$$
\varepsilon: \operatorname{int}(\hat{N}) \rightarrow(0, \infty)
$$

be a continuous function. Apply Proposition 13 to obtain an ambient isotopy $\beta_{t}$ of int $(\hat{N})$ such that $\beta_{1} \circ G$ int $(\hat{N})$ is piecewise-linear and

$$
\text { distance }\left(\beta_{t}(x), x\right)<\varepsilon(x) \text { for all } x \in \operatorname{int}(\hat{N}) \text {. }
$$

Let $Z=N\left(H, M^{\prime \prime}\right)$, and for $j \geqq 3$, define

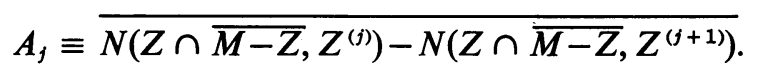

Since $g \mid \overline{M-H}$ is simplicial, $g\left(A_{j}\right)$ is a subcomplex of $Q^{(j+1)}$. Let

$$
B_{j} \equiv N\left(A_{j}, Z^{(j+3)}\right) \quad \text { and } \quad C_{j} \equiv N\left(g\left(A_{j}\right), Q^{(j+3)}\right) .
$$

Thus $g(M) \cap C_{j}=g\left(B_{j}\right)$. The intersection $C_{j} \cap C_{w}=\varnothing$ (unless $w=j-1, j, j+1$ ). By Theorem 11, there is a $\delta_{2 j+1}$ such that for any piecewise-linear embedding $g^{2 j+1}: B_{2 j+1} \rightarrow C_{2 j+1}$ that is equal to $g \mid B_{2 j+1}$ except possibly on $A_{2 j+1}$, that maps $A_{2 j+1}$ into int $\left(C_{2 j+1}\right)$, and that is within $\delta_{2 j+1}$ of $g$ on $A_{2 j+1}$, then there is a piecewise-linear ambient isotopy $\Omega_{t}^{2 j+1}$ of $C_{2 j+1}$ that fixes bdy $\left(C_{2 j+1}\right)$, that satisfies

$$
\Omega_{1}^{2 j+1} \circ g^{2 j+1}=g \mid B_{2 j+1},
$$

and that moves points less than minimum $\{1 / 2 j+1, \varepsilon / 2\}$. Also by Theorem 11, there is a $\delta_{2 j}$ such that if

$$
g^{2 j}: A_{2 j} \rightarrow \operatorname{int}\left(C_{2 j}\right),
$$

and $g^{2 j}$ is within $\delta_{2 j}$ of $g \mid A_{2 j}$, then there is a piecewise-linear ambient isotopy $\Omega_{t}^{2 j}$ of $C_{2 j}$ that fixes bdy $\left(C_{2 j}\right)$, that satisfies

$$
\Omega_{1}^{2 j} \circ g^{2 j}=g \mid A_{2 j},
$$

and that moves points less than

$$
\text { minimum }\left\{\frac{1}{2 j}, \frac{\varepsilon}{2}, \frac{\delta_{2 j+1}}{2}, \frac{\delta_{2 j-1}}{2}, \frac{\operatorname{mesh}\left(C_{2 j+1}^{(2 j+4)}\right)}{2}, \frac{\operatorname{mesh}\left(C_{2 j-1}^{(2 j+2)}\right)}{2}\right\} \text {. }
$$

Let $\varepsilon(x)$ : int $(\hat{N}) \rightarrow(0, \varepsilon / 2)$ be any continuous function that goes to zero as $x$ goes to bdy $(\hat{N})$, and such that

$$
\varepsilon(x)<\operatorname{minimum}\left\{\operatorname{mesh}\left(C_{f}^{(j+3)}\right) / 2, \delta_{j} / 2\right\}
$$

(if $x \in C_{j}$ ). Let $\beta_{t}$ be the ambient isotopy of int $(\hat{N})$ defined above. The behavior of $\varepsilon(x)$ near the boundary of $N$ implies that $\beta_{t}$ extends by the identity to an ambient isotopy on $Q$. The homeomorphism $\beta_{1} \circ g$ is piecewise-linear except at $Z \cap \overline{M-Z}$. Take the map $g^{2 j}$ discussed above to be $\beta_{1} \circ g \mid A_{2 j}$. Then since $C_{2 j} \cap C_{2 w}=\varnothing$ if $j \neq w$, the composition $\Omega_{t}^{\text {even }}$ of the $\Omega_{t}^{2 j}$ is well defined. Since each $\Omega_{t}^{2 j}$ moves points less than $1 / 2 j$, $\Omega_{t}^{\text {even }}$ extends by the identity to an ambient isotopy on $Q$. The 
homeomorphism $\Omega_{1}^{\text {even }} \circ \beta_{1} \circ g$ is piecewise-linear except at $Z \cap \overline{M-Z}$, and equals $g$ on $\overline{M-Z} \bigcup_{j=2}^{\infty} A_{2 j}$.

Let $\Omega_{1}^{\text {even }} \circ \beta_{1} \circ g \mid B_{2 j+1}$ be the map $g^{2 j+1}$ discussed above. Again the composition $\Omega_{t}^{\text {odd }}$ of the $\Omega_{t}^{2 j+1}$ is well defined and extends by the identity to an ambient isotopy on $Q$. Define $\Omega_{t}=\Omega_{t}^{\text {odd }} \circ \Omega_{t}^{\text {even }} \circ \beta_{t}$. Then $\Omega_{t}$ is an ambient isotopy of $Q$ that moves points less than $\varepsilon$, and such that $\Omega_{1} \circ g$ is piecewise-linear except possibly at $Z \cap \overline{M-Z}$; but $\Omega_{1} \circ g$ is piecewise-linear there since

$$
\Omega_{1} \circ g\left|\overline{M-Z} \bigcup_{j=3}^{\infty} A_{j}=g\right| \overline{M-Z} \bigcup_{j=3}^{\infty} A_{j},
$$

and $g$ is piecewise-linear on $\overline{M-Z} \bigcup_{j=3}^{\infty} A_{j}$. Consequently, $\Omega_{1} \circ g$ is piecewiselinear on $M$. This completes the proof of Theorem 14 .

\section{REFERENCES}

1. R. H. Bing, Radial engulfing, Conference on the Topology of Manifolds (Michigan State Univ., E. Lansing, Mich., 1967) Prindle, Weber \& Schmidt, Boston, Mass., 1968, pp. 1-18. MR 38 \#6560.

2. R. H. Bing and J. M. Kister, Taming complexes in hyperplanes, Duke Math. J. 31 (1964), 491-511. MR 29 \#1626.

3. E. H. Connell, Approximating stable homeomorphisms by piecewise-linear ones, Ann. of Math. (2) 78 (1963), 326-338. MR 27 \#4238.

4. V. K. Gugenheim, Piecewise linear isotopy and embedding of elements and spheres. I, Proc. London Math. Soc. (3) 3 (1953), 29-53. MR 15, 336.

5. R. C. Kirby, Smoothing locally flat imbeddings of differentiable manifolds, Topology 6 (1967), 207-220. MR 35 \#2291.

6. R. C. Kirby and L. C. Siebenmann, A proof of the stable homeomorphism theorem, Bull. Amer. Math. Soc. (to appear).

7. W. B. R. Lickorish, The piecewise linear unknotting of cones, Topology 4 (1965), 67-91. MR 34 \#3585.

8. R. T. Miller, Close isotopies on piecewise linear manifolds, Ph.D. Thesis, University Microfilms, Ann Arbor, Michigan, 1968.

9. E. C. Zeeman, Seminar on combinatorial topology, Inst. Hautes Études Sci., Paris, 1963 (Mimeographed notes).

UNIVERSity OF ChicAGo,

Chicago, Illinois 60637 